\title{
Oral Toxicity Study and Skin Sensitization Test of a Cricket
}

\author{
Hyeon Yeol Ryu ${ }^{1, \dagger}$, Somin Lee ${ }^{1,2, \dagger}$, Kyu Sup Ahn', Hye Jin Kim ${ }^{1}$, Sang Sik Lee', Hyuk Ju Ko', Jin Kyu Lee', \\ Myung-Haing $\mathrm{Cho}^{2}$, Mi Young Ahn ${ }^{3}$, Eun Mi Kim${ }^{4}$, Jeong Ho $\mathrm{Lim}^{4}$ and Kyung Seuk Song ${ }^{1}$ \\ ${ }^{1}$ Toxicity Evaluation Center, Korea Conformity Laboratories (KCL), Incheon, Korea \\ ${ }^{2}$ Laboratory of Toxicology, College of Veterinary Medicine, Seoul National University, Seoul, Korea \\ ${ }^{3}$ Department of Agricultural Biology, National Academy of Agricultural Science (RDA), Wanju, Korea \\ ${ }^{4}$ Korea Food Research Institute, Seongnam, Korea
}

(Received November 3, 2015; Revised December 29, 2015; Accepted January 27, 2016)

\begin{abstract}
Crickets have been attracting considerable interest in the field of nutrition and toxicology due to the global exhaustion of food resulting from a growing population. The cricket is normally eaten in several countries after roasting, similar to the grasshopper; however, safety evaluation data on cricket powder is limited. Here, we performed general toxicity studies of cricket powder including a single, 2-week repeated dose range evaluation test, a 13-week repeated oral dose toxicity test in Sprague-Dawley rats, a single oral dose toxicity test in Beagle dogs, and a skin sensitization test in guinea pigs following the Organization for Economic Cooperation and Development test guidelines 406 and 408 in addition to Good Laboratory Practice. To investigate the NOAEL and target organs of cricket powder, Sprague-Dawley rats were allocated to 4 groups: vehicle control, 1,250 mg/kg, 2,500 mg/kg, 5,000 mg/kg dose test groups and cricket powder was administered over 13 weeks after single dose and dose range finding studies in rats based on the results of the single oral administration toxicity study in rats and Beagle dogs. The results of the study showed that the NOAEL of cricket powder was over $5,000 \mathrm{mg} / \mathrm{kg}$ for both sexes of rats without adverse effects in a 13-week repeated oral toxicity study and there was no skin hypersensitivity reaction. Therefore, our results reveal that crickets can be widely used as a new substitute food or nutrient resource.
\end{abstract}

Key words: Cricket, Gryllus bimaculatus, Oral dose toxicity, Skin sensitization, NOAEL

\section{INTRODUCTION}

According to recent studies, crickets are used as a traditional treatment for fever and hypertension. There has been a great deal of research on the use of crickets as a new substitute food. Crickets contain a large amount of high quality protein, ash, moisture, and essential fatty acids such as oleic, linoleic, and r-linoleic acid (1). As a result of the analytical analysis of cricket powder, it also contains several minerals including $\mathrm{Ca}(0.25 \%), \mathrm{P}(0.85 \%), \mathrm{K}(0.98 \%), \mathrm{Mg}$ (0.11\%), Mn (68 ppm), Fe (76 ppm), Zn (283 ppm), Cu (12

Correspondence to: Kyung Seuk Song, Toxicity Evaluation Center, Korea conformity laboratories, Incheon 406-840, Korea E-mail: songks@kcl.re.kr

${ }^{\dagger}$ These authors contributed equally to this work.

This is an Open-Access article distributed under the terms of the Creative Commons Attribution Non-Commercial License (http:// creativecommons.org/licenses/by-nc/3.0) which permits unrestricted non-commercial use, distribution, and reproduction in any medium, provided the original work is properly cited. ppm). In addition, crickets as part of the diet can lower the blood ethanol level by increasing liver mitochondrial alcohol metabolizing enzymes such as alcohol dehydrogenase $(\mathrm{ADH})$ and acetaldehyde dehydrogenase (ALDH). Many studies have revealed the potential of crickets as a health supplement. The glycosaminoglycan (GAG) mediating the anti-atherosclerotic and antilipemic effects, have been investigated in cricket feeding Wistar rats (2) and cricket extracts were studied as a therapeutic agent for inflammatory diseases such as chronic arthritis (3). The acute toxicity of crickets in Sprague-Dawley (SD) rats was tested and cricket was found to be non-toxic with an oral $\mathrm{LD}_{50}$ value of $>5,000 \mathrm{mg} / \mathrm{kg}$ (4). These unique characteristics of crickets have motivated the toxicological tests herein including a single, 2-week repeated test (dose range finding, DRF), a 13-week repeated oral dose toxicity test in SD rats, a single oral dose-escalation toxicity test in Beagle dogs and a skin sensitization test (Buehler method) in Hartley guinea pigs. Using these in vivo tests, we determined that crickets cause no toxicological issues as part of the diet and can serve as an excellent food source, resolving the scarcity of food, in 
addition to being a possible health supplement in the future.

\section{MATERIALS AND METHODS}

Preparation of test materials. Crickets, specifically Gryllus bimaculatus (G. bimaculatus), were processed by the Korean Food Research Institute (KFRI) in Gyeonggido, South Korea. The cricket sample was washed 3 times with distilled water to eliminate impurities, completely dehydrated and freeze-dried at $-70^{\circ} \mathrm{C}$ using a Freeze Dryer Bondiro FD8508 (Ilshin Lab. Co., Yangju, Korea). $50 \mathrm{~g}$ of the sample was homogenized and passed through an 80 mesh net. The general components (moisture, crude ash, crude protein, crude fat and crude fiber), free fatty acid content, minerals, percentage of amino acid composition and heavy metal components were analyzed by a food analysis research center in KFRI. According to the AOAC 2002, the general component analyses were conducted depending on the sample types: moisture was measured using the $105^{\circ} \mathrm{C}$ air-oven method, crude ash was measured using the $550^{\circ} \mathrm{C}$ dry ashing method, crude protein was measured using a Kjeltec auto sampler system 8440 analyzer (Foss Tecator AB, Höganäs, Sweden), crude fat was measured using the soxhlet extraction method, the free fatty acid content, minerals, percentage of amino acid composition, and heavy metal component were analyzed following the AOAC methods.

Animals. Methods were approved by the animal care and use committee at the Korea conformity laboratory (IA13-00701, IA14-00003, IA14-00312 and IA14-00043). All experiments were designed and conducted under the Organization for Economic Cooperation and Development (OECD) test guidelines No. 406 (5) and 408 (6), Good Laboratory Practice (GLP) and, the Korean Ministry of Food and Drug Safety (KMFDS) notice no. 2013-121 'Toxicity test standards of medicine and medical supplies'. 5- to 7week-old SD rats, 5- to 6-month-old beagle dogs and 4- to 5 -week-old Hartley guinea pigs were delivered prior to the administration. All animals were obtained from ORIENTBIO (Sungnam, Korea). Animals were maintained in a laboratory facility at a standard temperature of $23 \pm 2{ }^{\circ} \mathrm{C}$ and a relative humidity of $50 \pm 10 \%$ under a $12: 12 \mathrm{hr}$ light/dark cycle. Rats were fed a rodent diet (Harlan Teklad, Madison, WI, USA). Beagle dogs were offered a daily ration of lab canine chow (Cargill Agri Purina, Inc., Seongnam, Korea). Guinea pigs were fed a Guinea pig diet (Cargill Agri Purina, Inc.). All animals were provided with tap water purified by a reverse osmosis filtering system ad libitum.

Single oral dose toxicity study in SD rats. When the rats became 8 weeks old, they were administered the test substances by gavage. Individual dosing volumes were calculated based on $20 \mathrm{~mL} / \mathrm{kg}$ body weight. SD rats were ran- domly allocated into 4 groups per 5 animals each: vehicle control, 1,250, 2,500 and 5,000 mg/kg test groups. Each animal was observed for mortality, clinical signs, and behavioral changes after the first half-hour, followed by every hour for six hours after test substance administration and once daily thereafter for 13 days. Individual animal weights were recorded at acquisition, grouping, immediately before administration, once a week during the study and immediately before necropsy. On day 13 , surviving animals were anesthetized with $\mathrm{CO}_{2}$ inhalation and the abdominal aorta and vena cava were severed. Vital organs were carefully observed macroscopically.

A 2-week repeated DRF study and a 13-week repeated oral dose toxicity study in SD rats. In the 2-week repeated DRF study, 5-week-old male and female specificpathogen free SD rats were acclimated for 5 days before administration. When the rats became 6-weeks-old, they were exposed to cricket powder by gavage for 14 days. Dose levels were chosen based on the observation of the single dose oral toxicity study. Individual dosing volumes were calculated based on $20 \mathrm{~mL} / \mathrm{kg}$ body weight. During the study, mortality, clinical signs, body weight, food consumption, ophthalmology, urinalysis, hematology, plasma coagulation, blood biochemistry, organ weight, necropsy, and histopathological evaluations were conducted. Based on the test results, the dose for the 13-week repeated toxicity study was determined.

5-week-old male and female SD rats were acclimated for 7 days before test substance administration. The rats were randomly allocated into 4 groups per 10 animals each: vehicle control, 1,250, 2,500 and 5,000 mg/kg/day treated groups. Dose levels were chosen based on the observation of the 2week repeated DRF study. When the rats became 6 weeks old, they were exposed to cricket powder by gavage for 90 days of oral administration. Clinical signs and mortality for all animals were monitored once a day. Body weight and food consumption were monitored once a week. The administered dose was calculated based on weight changes. Urine samples were collected randomly from 5 animals in each group using metabolic cages in the last week of the administration period. The urine samples were analyzed for the test items below using urine test strips (SIEMENS, Erlangen, Germany) and the urine auto-analyzer, CliniTek 50 (SIEMENS). Urine colors were observed by the naked eye. The blood was analyzed using the Hematology analyzer, ADVIA 2120 (SIEMENS). Animals were fasted overnight and anesthetized with $\mathrm{CO}_{2}$. Blood samples were collected from the abdominal aorta at necropsy. EDTA-2K was used as an anticoagulant. A plasma coagulation test was conducted to analyze PT (prothrombin time) and APTT (active partial thromboplastin time) using the blood coagulation analyzer, ACL7000 (Instrumentation Laboratory Co., Lexington, MA, USA). Blood samples were collected from 
the abdominal aorta at necropsy. 3.2\% sodium citrate solution was used as an anticoagulant. Serum biochemistry analysis was conducted using the biochemistry analyzer, Hitachi7180 (Hitachi, Tokyo, Japan). Serum was collected by centrifugation of the blood at 3,000 rpm for $10 \mathrm{~min}$. Animals in the vehicle control and $5,000 \mathrm{mg} / \mathrm{kg} /$ day treated groups were subjected to eye examinations in the last week of the administration period. Animals were terminated by exsanguination and subjected to a full necropsy with complete post-mortem examinations. At necropsy, testis, prostate, epididymis, seminal vesicle, ovary, uterus, bladder, spleen, liver, thymus, thyroid gland, adrenal gland, kidney, heart, tongue, esophagus, trachea, lung, brain, and pancreas were carefully removed and weighed using an electronic balance. Testes and epididymides were fixed in Bouin's solution and eyeballs were fixed in Davidson's solution. Other organs were fixed in $10 \%$ neutral buffered formalin. Thereafter, samples were embedded in paraffin, and sectioned with microtome. Sample slides were stained with hematoxylin and eosin.

\section{Single oral dose-escalation toxicity in Beagle dogs.} On arrival, the external appearance of the animals was examined based on the certificate of vaccination provided by the supplier. Healthy animals were acclimated for 20 days in the animal room where the present study was performed. During the acclimation period, clinical signs were monitored once a day and healthy animals were used in the main study.

In the 2-week repeated DRF study in SD rats, there was no toxicological effect in the $1,250,2,500$, or $5,000 \mathrm{mg} / \mathrm{kg}$ test groups. Based on these results, 312.5 and $625 \mathrm{mg} / \mathrm{kg}$ were selected as a first administration dosage, followed by 1,250 and $2,500 \mathrm{mg} / \mathrm{kg}$ as a second administration dosage. Dogs of each sex were fasted for one night before dosing. Gelatin capsules were placed on the rear part of the tongue and the throat was massaged with smooth strokes to facilitate swallowing. The test substance was administered once a day at 4-day intervals, twice in total in the same animals. Clinical signs and mortality were monitored continuously for the first hour following administration and every hour thereafter for $6 \mathrm{hrs}$. During the entire experimental period, animals were observed daily. Individual animals were weighed before administration and at days 1 , 3 , and 6 after administration. On day 14 following the second administration, all animals were anesthetized with Entobar (Hanlim Pharmacy, Yongin, Korea) and then terminated by exsanguination of the axillary artery and vein. Complete post-mortem examinations were performed on all vital organs.

Skin sensitization test (Buehler method) in Hartley guinea pigs. Animals were acclimated for 13 days, and those with the best appearance were selected for testing.
Animals were accepted based on the certification provided by the supplier. Animals were weighed one day before the administration and were randomly assigned to the test or control groups. In the pilot study, $20 \%(\mathrm{w} / \mathrm{v})$ was selected as the highest concentration based on a solubility test. None of the animals in the $20 \%$ test substance-treated group showed skin irritation, therefore, in the main study, the highest concentration $(20 \%)$ was determined as the induction and challenge dose. The induction dose should cause mild irritation and the challenge dose should be the highest non-irritating dose. For the positive substance, $\alpha$-Hexyl Cinnamic Aldehyde, the induction and challenge doses were $90 \%$ and $50 \%$ corn-oil, respectively (7).

The guinea pigs were divided into 3 groups: 5 animals each for the vehicle and positive control groups and 10 animals for the test group. Before the test substance administration, the right flank of all tested animals was cleared of hair (closely-clipped). The test substance and vehicle were fully loaded into cotton patches $\left(4 \sim 6 \mathrm{~cm}^{2}, 0.4 \mathrm{~mL}\right)$ on days 0,7 , and 14, and applied to the lower dorsal part of the flank ( $2^{\text {nd }}$ administration site) using a non-irritant adhesive vinyl Tegaderm ${ }^{\mathrm{TM}} 1624 \mathrm{~W}$ (3M, St. Paul, MN, USA). The test patch was held in contact with the skin by Micropore ${ }^{\mathrm{TM}}$ 1530-1 surgical tape (3M) for 6 hrs. For the positive control, HCA was applied to the test area using the same method. Challenge exposure was performed in all animals on day 28. The left side of the flank (a different site from the induction phase) was cleared of hair in all animals. Distilled water was applied to the left anterior flank of the vehicleand test substance-treated groups and the left posterior flank of the animals was treated with the test substance and vehicle. For the positive control, HCA was applied to the left posterior flank of the animals and corn oil was applied to left anterior flank using the same method. Clinical signs and mortality were monitored once a day up to the final challenge observation. Body weight was measured on the first day of acclimation, at grouping, on the $1^{\text {st }}, 2^{\text {nd }}$, and $3^{\text {rd }}$ inductions, at the challenge, at 24 and $48 \mathrm{hr}$ after removal of the challenge patch. Approximately $21 \mathrm{hr}$ after removing the patch, the challenge area was cleared of hair, and three hours later, the skin reactions were observed and recorded. Skin reactions were evaluated according to the Magnusson and Kligman grading scale offered at KMFDS. Based on the score, the sensitization rate $(\%)$ was calculated as follows (Number of animals with a positive reaction/number of tested animals) $\times 100$.

Statistical analysis. The differences among the vehicle control and the all dosing groups were analyzed through the parametric multiple comparison procedures or non-parametric multiple comparison procedures. It was determined to be statistically significant if $p<0.05$. Statistical significance was represented by percentage. SPSS for Windows version 12.0 software (SPSS, Chicago, IL, U.S.A.) was 
used for analysis. Asterisks (*) indicate statistically significant differences compared with the control groups. 1) Analysis of continuous data (body weights, food consumption, water consumption, hematology, blood biochemistry, organ weights): The statistical treatment was conducted to suppose the normality. The differences among the groups were examined and certificated the equal variance through the standard one-way analysis of variance (ANOVA). If the test showed statistical significance, the data was analyzed through the parametric multiple comparison to compare the vehicle control group with the experimental groups. If the equal variance was admitted, Duncan's test was used and if the equal variance was not admitted, Dunnett's T-test was applied. 2) Analysis of non-continuous data (urinalysis): The data was converted by scale conversion and then analyzed by Chi-squared analysis.

\section{RESULTS}

Nutritional content of cricket powder. The nutritional content of cricket powder was analyzed. The nutritional compositions of cricket are listed in Table 1. The

Table 1. Component analysis of cricket powder

\begin{tabular}{|c|c|c|c|c|}
\hline \multicolumn{5}{|c|}{ General component (unit: $\mathrm{mg} / 100 \mathrm{mg}$, d.b.) } \\
\hline Moisture & Crude ash & Crude protein & Crude fat & Crude fiber \\
\hline $0.88 \pm 0.09$ & $7.69 \pm 0.08$ & $68.37 \pm 0.04$ & $16.03 \pm 0.56$ & $10.92 \pm 0.19$ \\
\hline \multicolumn{5}{|c|}{ Free fatty acid (unit: \%) } \\
\hline \multicolumn{2}{|c|}{ Componets } & Contents & Componets & Contents \\
\hline \multicolumn{2}{|c|}{ C14:0 (Myistic acid) } & ND & Others & $6.38 \pm 0.69$ \\
\hline \multicolumn{2}{|c|}{ C16:0 (Palmitic acid) } & $21.00 \pm 1.13$ & Unsaturated fatty acid & $63.55 \pm 0.56$ \\
\hline \multicolumn{2}{|c|}{ C16:1 (Pamitoleic acid) } & ND & Saturated fatty acid & $30.07 \pm 0.96$ \\
\hline \multicolumn{2}{|c|}{ C18:0 (Stearic acid) } & $9.07 \pm 1.74$ & Total & 100 \\
\hline \multicolumn{2}{|c|}{ C18:1n9c (Oleic acid) } & $20.40 \pm 1.18$ & & \\
\hline \multicolumn{2}{|c|}{ C18:2n6c (Linoleic acid) } & $40.70 \pm 1.94$ & & \\
\hline \multicolumn{2}{|c|}{ C20:1 (cis-11-Eicosenoic acid) } & ND & & \\
\hline \multicolumn{2}{|c|}{ C18:3n3 (Linolenic acid) } & $2.45 \pm 0.25$ & & \\
\hline \multicolumn{2}{|c|}{ C20:2 (cis-11,14-Eicosadienic acid) } & ND & & \\
\hline \multicolumn{5}{|c|}{ Mineral } \\
\hline \multicolumn{2}{|l|}{ Component } & & Component & Contents \\
\hline \multicolumn{2}{|l|}{$\mathrm{Ca}(\%)$} & & Mn (ppm) & 68 \\
\hline \multicolumn{2}{|l|}{$\mathrm{P}(\%)$} & & $\mathrm{Fe}(\mathrm{ppm})$ & 76 \\
\hline $\mathrm{K}(\%)$ & & & $\mathrm{Zn}(\mathrm{ppm})$ & 283 \\
\hline $\operatorname{Mg}(\%)$ & & & $\mathrm{Cu}(\mathrm{ppm})$ & 12 \\
\hline & & Amino acid (unit & & \\
\hline Component & & & Component & Contents \\
\hline Aspartic acid & & & Tyrosine & 3.16 \\
\hline Threonine & & & Phenylalanine & 2.24 \\
\hline Serine & & & Lysine & 3.51 \\
\hline Glutamic acid & & & Histidine & 1.43 \\
\hline Glycine & & & Arginine & 3.9 \\
\hline Alanine & & & Cysteine & 0.57 \\
\hline Valine & & & Methionine & 0.96 \\
\hline Isoleucine & & & Proline & 3.56 \\
\hline Leucine & & & & \\
\hline & & eavy metal (unit: & & \\
\hline & ponent & & Contents & \\
\hline & As & & ND & \\
\hline & $\mathrm{Cd}$ & & 0.03 & \\
\hline & $\mathrm{Hg}$ & & 1.1 & \\
\hline & $\mathrm{Pb}$ & & ND & \\
\hline
\end{tabular}

The results are mean \pm standard deviation (SD). ND: not detected. 


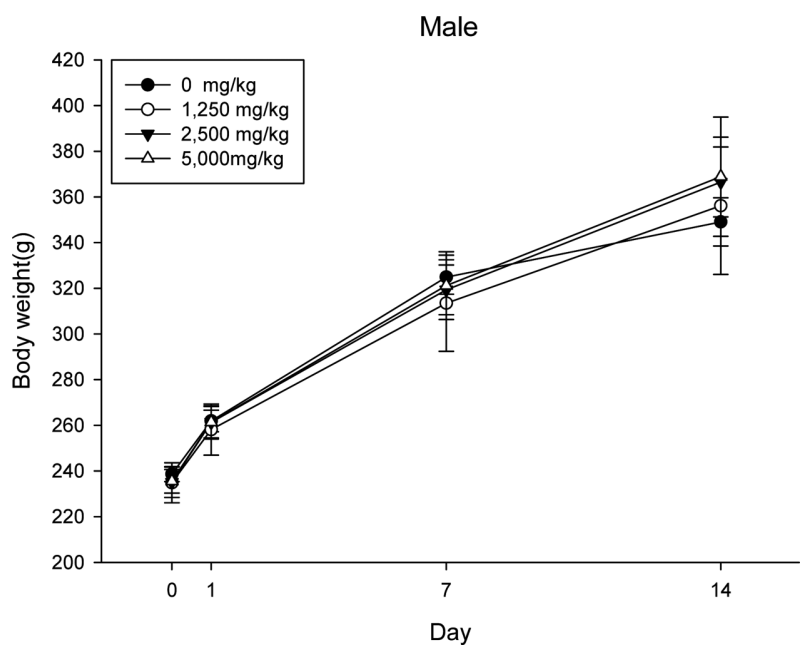

Fig. 1-1. Body weight changes in male rats of single oral dose toxicity study. Body weight changes of male rats of vehicle control and cricket powder treated group ( $\mathrm{n}=10$ per each groups). Error bar represents standard deviation.

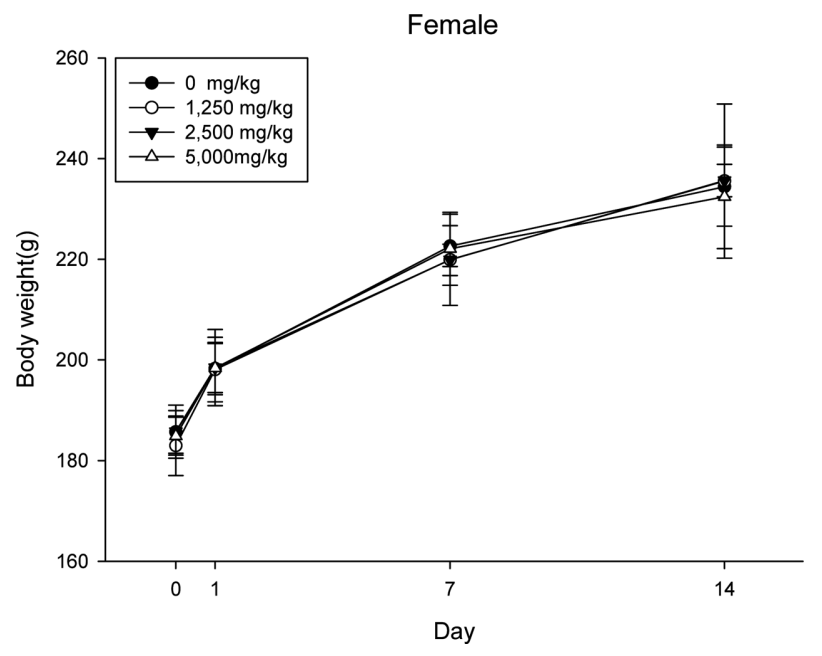

Fig. 1-2. Body weight changes in female rats of single oral dose toxicity study. Body weight changes of female rats of vehicle control and cricket powder treated group $(n=10$ per each groups). Error bar represents standard deviation.

Table 2. Body weight gains and clinical signs of Beagle dogs at the $1^{\text {st }}$ and $2^{\text {nd }}$ administration of cricket powder

\begin{tabular}{|c|c|c|c|c|c|}
\hline \multicolumn{6}{|c|}{ Mortalities and clinical signs } \\
\hline \multicolumn{3}{|c|}{ Male } & \multicolumn{3}{|c|}{ Female } \\
\hline Groups (mg/kg) & Weight gain & Signs & Groups (mg/kg) & Weight gain & Signs \\
\hline 312.5 & $0.14^{\mathrm{a}}$ & Normal & 312.5 & $0.12^{\mathrm{a}}$ & Normal \\
\hline 625 & $0.06^{\mathrm{a}}$ & Normal & 625 & $0.14^{\mathrm{a}}$ & Normal \\
\hline 1,250 & $0.76^{\mathrm{b}}$ & Normal & 1,250 & $0.48^{\mathrm{b}}$ & Normal \\
\hline 2,500 & $0.72^{\mathrm{b}}$ & Normal & 2,500 & $0.56^{\mathrm{b}}$ & Normal \\
\hline
\end{tabular}

Only one animal per each group was examined. a: weight gains between day 0 and day 1 at the $1^{\text {st }}$ administration, b: weight gain between day 0 and day 14 at the $2^{\text {nd }}$ administration.

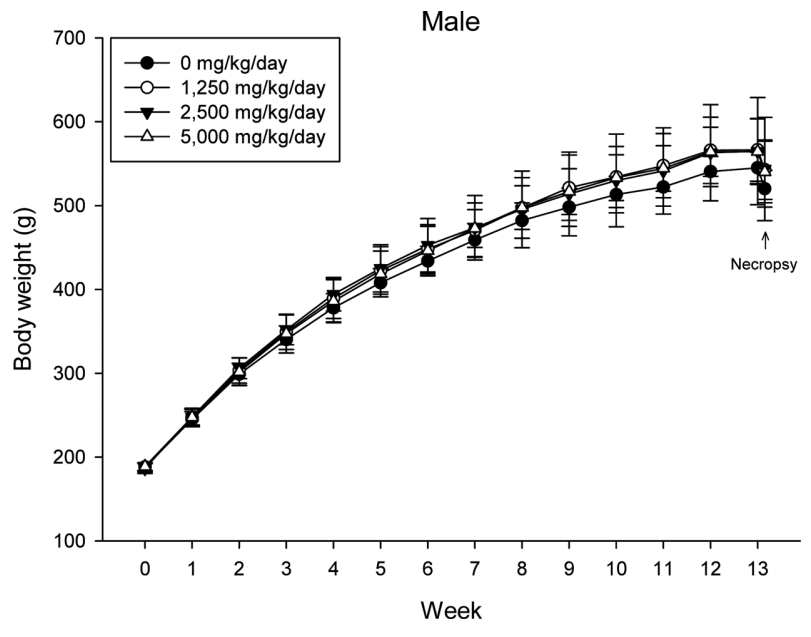

Fig. 2-1. Body weight changes in male rats in 13-week repeated oral dose toxicity study. Body weight changes of male rats of vehicle control and cricket powder treated group $(n=10$ per each groups). Error bar represents standard deviation.

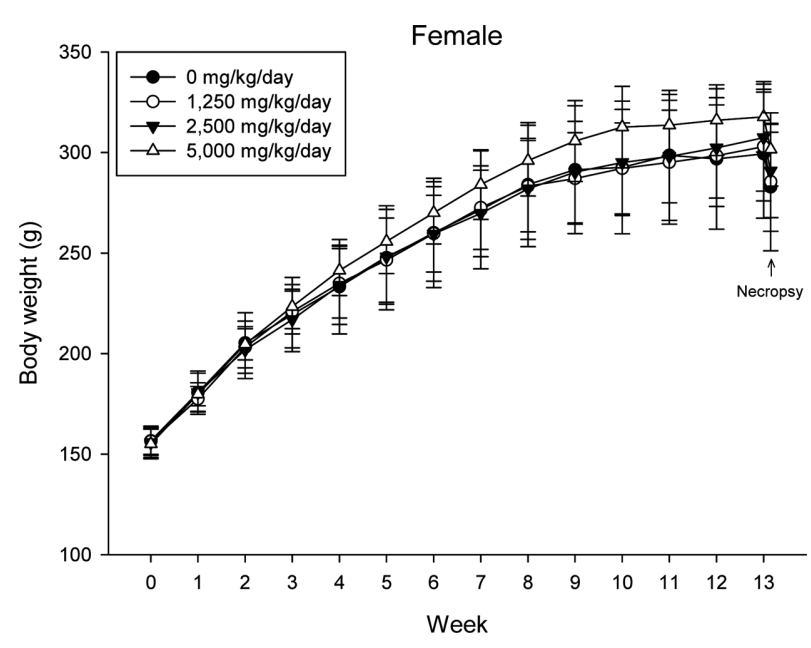

Fig. 2-2. Body weight changes in female rats in 13-week repeated oral dose toxicity study. Body weight changes of female rats of vehicle control and cricket powder treated group $(n=10$ per each groups). Error bar represents standard deviation. 
general components were found to be moisture $(0.88 \pm 0.09$ $\mathrm{mg} / 100 \mathrm{mg})$, crude ash $(7.69 \pm 0.08 \mathrm{mg} / 100 \mathrm{mg})$, crude protein $(68.37 \pm 0.04 \mathrm{mg} / 100 \mathrm{mg})$, crude fat $(16.03 \pm 0.56$ $\mathrm{mg} / 100 \mathrm{mg})$ and crude fiber $(10.92 \pm 0.19 \mathrm{mg} / 100 \mathrm{mg})$. With respect to the amount of free fatty acid, C18:2n6c (linoleic acid), which is an unsaturated fatty acid (USFA), was found to be an extremely substantial portion of the total free fatty acid $(40.70 \pm 1.94 \%)$. Regarding the amount of minerals, cricket powder was found to contain $0.205 \%$ calcium, $0.85 \%$ phosphate, $0.98 \%$ potassium, $0.11 \%$ magnesium, 68 ppm manganese, $76 \mathrm{ppm}$ iron, $283 \mathrm{ppm}$ zinc and $12 \mathrm{ppm}$ copper. In addition, cricket powder was found to have relatively even amounts of amino acids, with the exception of cysteine and methionine, which were much lower. A very small amount of heavy metals, 0.03 ppm cadmium and 1.1 ppm mercury, were detected.

Single oral dose toxicity study. No mortalities or notable clinical signs in the $0,1,250,2,500$ or $5,000 \mathrm{mg} / \mathrm{kg}$ test groups were observed. There was no toxicologically significant difference in the body weight changes in either the male or female test groups compared with the vehicle control group (Fig. 1-1, 1-2). At necropsy, there was no gross finding in either the male or female vehicle control groups compared with the test group. The approximate lethal dose (ALD) of cricket powder is considered greater than 5,000 $\mathrm{mg} / \mathrm{kg}$ body weight in rats.

Single oral dose-escalation toxicity in Beagle dogs. There were no clinical signs or mortalities observed during

Table 3. Urinalysis for the rats in the 13-week repeated oral dose toxicity study

\begin{tabular}{|c|c|c|c|c|c|c|c|c|c|}
\hline \multicolumn{10}{|c|}{ Summary of Urinalysis } \\
\hline \multirow{3}{*}{\multicolumn{2}{|c|}{ Test item }} & \multicolumn{4}{|c|}{ Male } & \multicolumn{4}{|c|}{ Female } \\
\hline & & \multicolumn{4}{|c|}{ Group (mg/kg/day) } & \multicolumn{4}{|c|}{ Group (mg/kg/day) } \\
\hline & & 0 & 1,250 & 2,500 & 5,000 & 0 & 1,250 & 2,500 & 5,000 \\
\hline Glucose & - & $5 / 5$ & $5 / 5$ & $5 / 5$ & $5 / 5$ & $5 / 5$ & $5 / 5$ & $5 / 5$ & $5 / 5$ \\
\hline Bilirubin & - & $5 / 5$ & $5 / 5$ & $5 / 5$ & $5 / 5$ & $5 / 5$ & $5 / 5$ & $5 / 5$ & $5 / 5$ \\
\hline \multirow{3}{*}{ Ketones" } & - & $4 / 5$ & $5 / 5$ & $3 / 5$ & $1 / 5$ & $5 / 5$ & $5 / 5$ & $4 / 5$ & $5 / 5$ \\
\hline & $+/-$ & $1 / 5$ & $0 / 5$ & $0 / 5$ & $4 / 5$ & $0 / 5$ & $0 / 5$ & $1 / 5$ & $0 / 5$ \\
\hline & $1+$ & $0 / 5$ & $0 / 5$ & $2 / 5$ & $0 / 5$ & $0 / 5$ & $0 / 5$ & $0 / 5$ & $0 / 5$ \\
\hline \multirow{6}{*}{ Specific gravity } & $\leq 1.005$ & $3 / 5$ & $3 / 5$ & $1 / 5$ & $0 / 5$ & $1 / 5$ & $4 / 5$ & $3 / 5$ & $1 / 5$ \\
\hline & 1.010 & $2 / 5$ & $1 / 5$ & $3 / 5$ & $1 / 5$ & $3 / 5$ & $0 / 5$ & $0 / 5$ & $0 / 5$ \\
\hline & 1.015 & $0 / 5$ & $1 / 5$ & $0 / 5$ & $2 / 5$ & $1 / 5$ & $1 / 5$ & $2 / 5$ & $2 / 5$ \\
\hline & 1.020 & $0 / 5$ & $0 / 5$ & $0 / 5$ & $2 / 5$ & $0 / 5$ & $0 / 5$ & $0 / 5$ & $1 / 5$ \\
\hline & 1.025 & $0 / 5$ & $0 / 5$ & $0 / 5$ & $0 / 5$ & $0 / 5$ & $0 / 5$ & $0 / 5$ & $1 / 5$ \\
\hline & $\geq 1.030$ & $0 / 5$ & $0 / 5$ & $1 / 5$ & $0 / 5$ & $0 / 5$ & $0 / 5$ & $0 / 5$ & $0 / 5$ \\
\hline Occult blood & - & $5 / 5$ & $5 / 5$ & $5 / 5$ & $5 / 5$ & $5 / 5$ & $5 / 5$ & $5 / 5$ & $5 / 5$ \\
\hline \multirow{6}{*}{$\mathrm{pH}$} & 6 & $0 / 5$ & $0 / 5$ & $0 / 5$ & $1 / 5$ & $0 / 5$ & $1 / 5$ & $0 / 5$ & $0 / 5$ \\
\hline & 6.5 & $0 / 5$ & $0 / 5$ & $0 / 5$ & $0 / 5$ & $0 / 5$ & $1 / 5$ & $2 / 5$ & $2 / 5$ \\
\hline & 7 & $0 / 5$ & $1 / 5$ & $2 / 5$ & $3 / 5$ & $1 / 5$ & $2 / 5$ & $1 / 5$ & $2 / 5$ \\
\hline & 7.5 & $0 / 5$ & $1 / 5$ & $1 / 5$ & $0 / 5$ & $1 / 5$ & $1 / 5$ & $1 / 5$ & $0 / 5$ \\
\hline & 8 & $3 / 5$ & $1 / 5$ & $2 / 5$ & $1 / 5$ & $2 / 5$ & $0 / 5$ & $1 / 5$ & $1 / 5$ \\
\hline & 8.5 & $2 / 5$ & $2 / 5$ & $0 / 5$ & $0 / 5$ & $1 / 5$ & $0 / 5$ & $0 / 5$ & $0 / 5$ \\
\hline \multirow{4}{*}{ Protein } & - & $3 / 5$ & $1 / 5$ & $0 / 5$ & $0 / 5$ & $3 / 5$ & $5 / 5$ & $4 / 5$ & $2 / 5$ \\
\hline & $+/-$ & $1 / 5$ & $2 / 5$ & $1 / 5$ & $1 / 5$ & $2 / 5$ & $0 / 5$ & $0 / 5$ & $1 / 5$ \\
\hline & $1+$ & $1 / 5$ & $2 / 5$ & $4 / 5$ & $3 / 5$ & $0 / 5$ & $0 / 5$ & $1 / 5$ & $1 / 5$ \\
\hline & $2+$ & $0 / 5$ & $0 / 5$ & $0 / 5$ & $1 / 5$ & $0 / 5$ & $0 / 5$ & $0 / 5$ & $1 / 5$ \\
\hline Urobilinogen & 0.2 & $5 / 5$ & $5 / 5$ & $5 / 5$ & $5 / 5$ & $5 / 5$ & $5 / 5$ & $5 / 5$ & $5 / 5$ \\
\hline Nitrate & - & $5 / 5$ & $5 / 5$ & $5 / 5$ & $5 / 5$ & $5 / 5$ & $5 / 5$ & $5 / 5$ & $5 / 5$ \\
\hline \multirow{2}{*}{ Leukocytes } & - & $5 / 5$ & $5 / 5$ & $4 / 5$ & $3 / 5$ & $5 / 5$ & $5 / 5$ & $5 / 5$ & $4 / 5$ \\
\hline & $+/-$ & $0 / 5$ & $0 / 5$ & $1 / 5$ & $2 / 5$ & $0 / 5$ & $0 / 5$ & $0 / 5$ & $1 / 5$ \\
\hline \multirow{2}{*}{ Color } & Colorless & $3 / 5$ & $1 / 5$ & $0 / 5$ & $0 / 5$ & $0 / 5$ & $1 / 5$ & $2 / 5$ & $0 / 5$ \\
\hline & Straw & $2 / 5$ & $4 / 5$ & $5 / 5$ & $5 / 5$ & $5 / 5$ & $4 / 5$ & $3 / 5$ & $5 / 5$ \\
\hline
\end{tabular}

Number of animals with the sign/Number of animals examined, -: Negative, +/-: Trace.

*: Significant difference among the groups, $p<0.01$. 
the test period and no body weight fluctuations (Table 2). There were no gross findings related to the test substance administration. NOAEL of cricket powder is considered greater than $2,500 \mathrm{mg} / \mathrm{kg}$ body weight in Beagle dogs.

2-week repeated DRF study and 13-week repeated oral dose toxicity study. During the 2-week repeated oral administration of $0,1,250,2,500$ or $5,000 \mathrm{mg} / \mathrm{kg}$ doses, the body weights of the male and female rats were comparable across the control and test groups (S Fig. 1-1, 1-2). Food consumption for each sex in the test groups was not significantly different compared with that of the vehicle control groups. There were no clinical signs or mortalities observed at the end of the experiment. With respect to the ophthalmoscopy results, there were no abnormalities detected in the test groups. In urinalysis, there was no significant difference between the test group and the vehicle control group in either sex (S Table 1). The result of the hematological anal-

Table 4. Urine sediment for the rats in the 13-week repeated oral dose toxicity study

\begin{tabular}{|c|c|c|c|c|c|c|c|c|c|}
\hline \multicolumn{10}{|c|}{ Summary of Urine Sediments } \\
\hline \multirow{3}{*}{ Test items } & \multirow{3}{*}{ Grade } & \multicolumn{4}{|c|}{ Male } & \multicolumn{4}{|c|}{ Female } \\
\hline & & \multicolumn{4}{|c|}{ Group (mg/kg/day) } & \multicolumn{4}{|c|}{ Group (mg/kg/day) } \\
\hline & & 0 & 1,250 & 2,500 & 5,000 & 0 & 1,250 & 2,500 & 5,000 \\
\hline $\mathrm{RBC}$ & 0 & $5 / 5$ & $5 / 5$ & $5 / 5$ & $5 / 5$ & $5 / 5$ & $5 / 5$ & $5 / 5$ & $5 / 5$ \\
\hline WBC & 0 & $5 / 5$ & $5 / 5$ & $5 / 5$ & $5 / 5$ & $5 / 5$ & $5 / 5$ & $5 / 5$ & $5 / 5$ \\
\hline Epithelial cell & 0 & $5 / 5$ & $5 / 5$ & $5 / 5$ & $5 / 5$ & $5 / 5$ & $5 / 5$ & $5 / 5$ & $5 / 5$ \\
\hline Casts & 0 & $5 / 5$ & $5 / 5$ & $5 / 5$ & $5 / 5$ & $5 / 5$ & $5 / 5$ & $5 / 5$ & $5 / 5$ \\
\hline
\end{tabular}

Number of animals with the sign/Number of animals examined.

Table 5-1. Hematological analysis for the male rats in the 13-week repeated oral dose toxicity study (Sex, Male)

\begin{tabular}{|c|c|c|c|c|}
\hline \multicolumn{5}{|c|}{ Summary of Hematological Values } \\
\hline \multirow{2}{*}{ Test item (unit) } & \multicolumn{4}{|c|}{ Group (mg/kg/day) } \\
\hline & 0 & 1,250 & 2,500 & 5,000 \\
\hline $\mathrm{WBC}^{1}(\mathrm{~K} / \mu \mathrm{L})$ & $9.76 \pm 1.71$ & $10.91 \pm 2.49$ & $8.58 \pm 2.42$ & $10.59 \pm 2.34$ \\
\hline $\mathrm{NE}^{2}(\mathrm{~K} / \mu \mathrm{L})$ & $1.57 \pm 0.59$ & $1.20 \pm 0.28$ & $1.11 \pm 0.30$ & $1.36 \pm 0.42$ \\
\hline $\mathrm{EO}^{3}(\mathrm{~K} / \mu \mathrm{L})$ & $0.08 \pm 0.03$ & $0.11 \pm 0.07$ & $0.07 \pm 0.02$ & $0.07 \pm 0.04$ \\
\hline $\mathrm{BA}^{4}(\mathrm{~K} / \mu \mathrm{L})$ & $0.00 \pm 0.00$ & $0.01 \pm 0.01$ & $0.01 \pm 0.01$ & $0.01 \pm 0.01$ \\
\hline $\mathrm{LY}^{5}(\mathrm{~K} / \mu \mathrm{L})$ & $7.80 \pm 1.19$ & $9.26 \pm 2.47$ & $7.07 \pm 2.10$ & $8.86 \pm 2.05$ \\
\hline $\mathrm{MO}^{6}(\mathrm{~K} / \mu \mathrm{L})$ & $0.21 \pm 0.09$ & $0.22 \pm 0.06$ & $0.18 \pm 0.07$ & $0.19 \pm 0.04$ \\
\hline $\operatorname{LUC}^{7}(\mathrm{~K} / \mu \mathrm{L})$ & $0.10 \pm 0.05$ & $0.11 \pm 0.06$ & $0.15 \pm 0.18$ & $0.11 \pm 0.07$ \\
\hline $\mathrm{NEP}^{8}(\%)$ & $15.7 \pm 3.8$ & $11.6 \pm 4.0$ & $13.3 \pm 3.8$ & $12.8 \pm 3.3$ \\
\hline $\operatorname{EOP}^{9}(\%)$ & $0.8 \pm 0.3$ & $1.0 \pm 0.7$ & $0.9 \pm 0.3$ & $0.7 \pm 0.5$ \\
\hline $\mathrm{BAP}^{10}(\%)$ & $0.0 \pm 0.0$ & $0.1 \pm 0.0$ & $0.1 \pm 0.1$ & $0.1 \pm 0.1$ \\
\hline $\operatorname{LYP}^{11}(\%)$ & $80.3 \pm 3.9$ & $84.2 \pm 4.2$ & $82.1 \pm 3.9$ & $83.6 \pm 3.2$ \\
\hline $\operatorname{MOP}^{12}(\%)$ & $2.2 \pm 0.8$ & $2.1 \pm 0.7$ & $2.1 \pm 0.6$ & $1.8 \pm 0.5$ \\
\hline $\operatorname{LUP}^{13}(\%)$ & $1.0 \pm 0.5$ & $1.0 \pm 0.5$ & $1.6 \pm 1.8$ & $1.0 \pm 0.5$ \\
\hline $\mathrm{RBC}^{14}(\mathrm{M} / \mu \mathrm{L})$ & $8.70 \pm 0.4$ & $8.80 \pm 0.47$ & $8.78 \pm 0.43$ & $8.85 \pm 0.48$ \\
\hline $\mathrm{Hb}^{15}(\mathrm{~g} / \mathrm{dL})$ & $15.5 \pm 0.8$ & $15.5 \pm 0.6$ & $15.3 \pm 0.6$ & $15.8 \pm 0.8$ \\
\hline $\mathrm{RDW}^{16}(\%)$ & $12.6 \pm 0.5$ & $13.1 \pm 1.0$ & $13.3 \pm 1.0$ & $12.5 \pm 0.5$ \\
\hline $\mathrm{HCT}^{17}(\%)$ & $45.9 \pm 2.4$ & $45.8 \pm 2.4$ & $45.6 \pm 2.0$ & $46.8 \pm 2.4$ \\
\hline $\mathrm{MCV}^{18}(\mathrm{fL})$ & $52.8 \pm 1.5$ & $52.1 \pm 2.4$ & $52.0 \pm 1.8$ & $53.0 \pm 2.5$ \\
\hline $\mathrm{MCH}^{19}(\mathrm{pg})$ & $17.9 \pm 0.5$ & $17.6 \pm 0.6$ & $17.5 \pm 0.6$ & $17.8 \pm 0.8$ \\
\hline $\operatorname{MCHC}^{20}(\mathrm{~g} / \mathrm{dL})$ & $33.8 \pm 0.5$ & $33.8 \pm 0.6$ & $33.6 \pm 0.5$ & $33.7 \pm 0.4$ \\
\hline $\operatorname{Reti}^{21}(\%)$ & $2.02 \pm 0.27$ & $2.32 \pm 0.65$ & $2.27 \pm 0.63$ & $2.02 \pm 0.59$ \\
\hline $\operatorname{PLT}^{22}(\mathrm{~K} / \mu \mathrm{L})$ & $985 \pm 97$ & $1127 \pm 138$ & $1091 \pm 130$ & $1081 \pm 159$ \\
\hline $\mathrm{MPV}^{23}(\mathrm{fL})$ & $6.1 \pm 0.7$ & $6.3 \pm 0.6$ & $6.4 \pm 0.5$ & $6.2 \pm 0.8$ \\
\hline
\end{tabular}

Mean \pm SD ( $n=10$ per each groups). 1: White blood cells, 2: Neutrophils, 3: Eosinophils, 4: Basophils, 5: Lymphocytes, 6: Monocytes, 7: Large unstained cells, 8: Percentage of neutrophils, 9: Percentage of eosinophils, 10: Percentage of basophils, 11: Percentage of lymphocytes, 12: Percentage of monocytes, 13: Percentage of large unstained cells, 14: Red blood cells, 15: Hemoglobin, 16: Red blood cell distribution width, 17: Hematocrit, 18: Mean corpuscular volume, 19: Mean corpuscular hemoglobin, 20: Mean corpuscular hemoglobin concentration, 21: Reticulocytes, 22: Platelets, 23: Mean platelet volume. 
ysis showed no significant difference in either sex in the test groups compared with the control group (S Table 2-1, 2-2). In serum biochemical analysis, triglyceride of male 1,250 $\mathrm{mg} / \mathrm{kg}$ test group and magnesium of female $1,250 \mathrm{mg} / \mathrm{kg}$ test group was statistically significantly increased compared with control group ( $p<0.01$ and $p<0.05$, respectively) (S Table 3-1, 3-2). We did not find any statistical significance in other test items in the test groups compared with the vehicle control group. In organ weights, absolute and relative organ weight of right ovary and spleen in female 5,000 $\mathrm{mg} / \mathrm{kg}$ test group was statistically significantly increased compared with the vehicle control group $(p<0.01$ and $p<$ 0.05 for absolute weight of right ovary and spleen, $p<0.01$ for relative weight of right ovary and spleen) (S Table 4-1, 4-2). However, there is no toxicological meaning on these findings: because the values were within the normal biological range and there was no dose-dependency. Under the study condition, there were not found target organs and systemic toxicological effects related with cricket powder in 2-week oral repeated DRF study. NOAEL was considered greater than $5,000 \mathrm{mg} / \mathrm{kg}$ body weight. Therefore, 5,000 $\mathrm{mg} / \mathrm{kg}$ dose was set as highest dose in 13-week repeated toxicity study.

During the 13-week repeated oral administration of 0 , $1,250,2,500$ and $5,000 \mathrm{mg} / \mathrm{kg}$ doses, the body weights of the male and female rats were comparable across the control and test groups (Fig. 2-1, 2-2). Food consumption for each sex in the test groups was not significantly different compared with that of the vehicle control groups. There were no clinical signs or mortalities observed at the end of the experiment. With respect to the ophthalmoscopy results, there were no abnormalities detected in the test groups. In urinalysis, there were no notable differences observed between the male and female groups in urinalysis except for ketone generation, which was statistically significantly increased in the male test substance-treated groups $(p<0.01)$ (Table 3). For urine sediment and volume analysis, there was no significant difference between the test group and the vehicle control group in either sex (Table 4).

Regarding hematological analysis, RBC and $\mathrm{Hb}$ concentrations were statistically significantly increased in the female $2,500 \mathrm{mg} / \mathrm{kg}$ test group compared with those in the vehicle

Table 5-2. Hematological analysis for the female rats in the 13-week repeated oral dose toxicity study (Sex, Female)

\begin{tabular}{|c|c|c|c|c|}
\hline \multicolumn{5}{|c|}{ Summary of Hematological Values } \\
\hline \multirow{2}{*}{ Test item (unit) } & \multicolumn{4}{|c|}{ Group (mg/kg/day) } \\
\hline & 0 & 1,250 & 2,500 & 5,000 \\
\hline $\mathrm{WBC}^{1}(\mathrm{~K} / \mu \mathrm{L})$ & $6.32 \pm 1.31$ & $6.03 \pm 1.38$ & $6.68 \pm 2.43$ & $7.56 \pm 2.41$ \\
\hline $\mathrm{NE}^{2}(\mathrm{~K} / \mu \mathrm{L})$ & $0.70 \pm 0.15$ & $0.75 \pm 0.22$ & $0.88 \pm 0.51$ & $0.86 \pm 0.34$ \\
\hline $\mathrm{EO}^{3}(\mathrm{~K} / \mu \mathrm{L})$ & $0.06 \pm 0.03$ & $0.06 \pm 0.02$ & $0.07 \pm 0.04$ & $0.06 \pm 0.03$ \\
\hline $\mathrm{BA}^{4}(\mathrm{~K} / \mu \mathrm{L})$ & $0.00 \pm 0.00$ & $0.00 \pm 0.00$ & $0.01 \pm 0.01$ & $0.01 \pm 0.01$ \\
\hline $\mathrm{LY}^{5}(\mathrm{~K} / \mu \mathrm{L})$ & $5.33 \pm 1.17$ & $5.01 \pm 1.31$ & $5.43 \pm 2.12$ & $6.39 \pm 2.11$ \\
\hline $\mathrm{MO}^{6}(\mathrm{~K} / \mu \mathrm{L})$ & $0.14 \pm 0.06$ & $0.13 \pm 0.07$ & $0.19 \pm 0.11$ & $0.16 \pm 0.06$ \\
\hline $\operatorname{LUC}^{7}(\mathrm{~K} / \mu \mathrm{L})$ & $0.10 \pm 0.05$ & $0.09 \pm 0.06$ & $0.09 \pm 0.06$ & $0.10 \pm 0.04$ \\
\hline $\mathrm{NEP}^{8}(\%)$ & $11.3 \pm 2.8$ & $13.0 \pm 5.3$ & $13.5 \pm 6.5$ & $11.7 \pm 3.3$ \\
\hline $\mathrm{EOP}^{9}(\%)$ & $0.9 \pm 0.5$ & $1.0 \pm 0.3$ & $1.1 \pm 0.5$ & $0.8 \pm 0.3$ \\
\hline $\mathrm{BAP}^{10}(\%)$ & $0.1 \pm 0.1$ & $0.1 \pm 0.1$ & $0.1 \pm 0.1$ & $0.1 \pm 0.1$ \\
\hline $\operatorname{LYP}^{11}(\%)$ & $84.2 \pm 2.9$ & $82.6 \pm 5.1$ & $81.2 \pm 6.0$ & $84.1 \pm 3.5$ \\
\hline $\operatorname{MOP}^{12}(\%)$ & $2.1 \pm 0.6$ & $2.1 \pm 0.9$ & $2.7 \pm 1.0$ & $2.1 \pm 0.5$ \\
\hline $\operatorname{LUP}^{13}(\%)$ & $1.4 \pm 0.6$ & $1.4 \pm 0.8$ & $1.3 \pm 0.7$ & $1.3 \pm 0.3$ \\
\hline $\mathrm{RBC}^{14}(\mathrm{M} / \mu \mathrm{L})$ & $7.90 \pm 0.32$ & $7.82 \pm 0.47$ & $8.37 \pm 0.30^{*}$ & $8.07 \pm 0.39$ \\
\hline $\mathrm{Hb}^{15}(\mathrm{~g} / \mathrm{dL})$ & $14.8 \pm 0.5$ & $14.8 \pm 0.7$ & $15.6 \pm 0.5^{*}$ & $15.1 \pm 0.6$ \\
\hline $\mathrm{RDW}^{16}(\%)$ & $11.1 \pm 0.2$ & $11.5 \pm 1.0$ & $11.2 \pm 0.4$ & $11.2 \pm 0.6$ \\
\hline $\mathrm{HCT}^{17}(\%)$ & $42.6 \pm 1.6$ & $42.6 \pm 2.2$ & $44.5 \pm 1.8$ & $43.4 \pm 1.6$ \\
\hline $\mathrm{MCV}^{18}(\mathrm{fL})$ & $53.9 \pm 1.2$ & $54.5 \pm 2.0$ & $53.2 \pm 2.2$ & $53.9 \pm 1.4$ \\
\hline $\mathrm{MCH}^{19}(\mathrm{pg})$ & $18.8 \pm 0.4$ & $18.9 \pm 0.7$ & $18.6 \pm 0.5$ & $18.7 \pm 0.5$ \\
\hline $\mathrm{MCHC}^{20}(\mathrm{~g} / \mathrm{dL})$ & $34.9 \pm 0.4$ & $34.7 \pm 0.5$ & $35.0 \pm 0.7$ & $34.7 \pm 0.8$ \\
\hline $\operatorname{Reti}^{21}(\%)$ & $1.86 \pm 0.32$ & $2.55 \pm 1.33$ & $1.77 \pm 0.35$ & $1.96 \pm 0.68$ \\
\hline $\mathrm{PLT}^{22}(\mathrm{~K} / \mu \mathrm{L})$ & $1073 \pm 123$ & $1071 \pm 144$ & $1045 \pm 95$ & $1132 \pm 105$ \\
\hline $\mathrm{MPV}^{23}(\mathrm{fL})$ & $5.6 \pm 1.1$ & $5.3 \pm 0.8$ & $5.5 \pm 1.0$ & $5.6 \pm 0.7$ \\
\hline
\end{tabular}

\footnotetext{
: Significant difference compared with control value, $p<0.05$.
}

Mean \pm SD ( $n=10$ per each groups). 1: White blood cells, 2: Neutrophils, 3: Eosinophils, 4: Basophils, 5: Lymphocytes, 6: Monocytes, 7: Large unstained cells, 8: Percentage of neutrophils, 9: Percentage of eosinophils, 10: Percentage of basophils, 11: Percentage of lymphocytes, 12: Percentage of monocytes, 13: Percentage of large unstained cells, 14: Red blood cells, 15: Hemoglobin, 16: Red blood cell distribution width, 17: Hematocrit, 18: Mean corpuscular volume, 19: Mean corpuscular hemoglobin, 20: Mean corpuscular hemoglobin concentration, 21: Reticulocytes, 22: Platelets, 23: Mean platelet volume. 
control group $(p<0.05)$ (Table 5-2). However, with respect to the other test criteria for the hematological analysis, we did not find any statistical significance in either sex in the test groups compared with the vehicle control group (Table $5-1,5-2)$. The results of the plasma coagulation tests showed no significant difference in either sex in the test groups compared with the control group (Table 6). In serum biochemical analysis, the total bilirubin of the female rats in the $1,250 \mathrm{mg} / \mathrm{kg}$ test group was statistically significantly increased $(p<0.05)$ (Table 7-2). However, with respect to the other test criteria of the serum biochemical analysis, we did not find any statistical significance in either sex in the

Table 6. Plasma coagulation values of the rats in the 13-week repeated oral dose toxicity study

\begin{tabular}{|c|c|c|c|c|}
\hline \multicolumn{5}{|c|}{ Summary of Plasma Coagulation Tests } \\
\hline Unit: sec & & & & \\
\hline \multirow{2}{*}{ Test items } & \multicolumn{4}{|c|}{ Group (mg/kg/day) } \\
\hline & 0 & 1,250 & 2,500 & 5,000 \\
\hline $\mathrm{PT}^{1}$ & $10.06 \pm 0.77$ & $10.30 \pm 0.79$ & $9.82 \pm 0.87$ & $9.93 \pm 0.47$ \\
\hline \multirow[t]{2}{*}{$\mathrm{APTT}^{2}$} & $18.0 \pm 4.8$ & $18.7 \pm 4.0$ & $17.9 \pm 3.9$ & $20.7 \pm 3.3$ \\
\hline & \multicolumn{4}{|c|}{ Sex: Female } \\
\hline \multirow{2}{*}{ Test items } & \multicolumn{4}{|c|}{ Group (mg/kg/day) } \\
\hline & 0 & 1,250 & 2,500 & 5,000 \\
\hline PT & $9.54 \pm 0.80$ & $9.48 \pm 0.56$ & $9.55 \pm 0.70$ & $9.56 \pm 0.72$ \\
\hline APTT & $16.8 \pm 2.4$ & $16.2 \pm 1.1$ & $16.0 \pm 1.4$ & $16.3 \pm 1.5$ \\
\hline
\end{tabular}

Mean \pm SD ( $n=10$ per each groups). 1: Prothrombin time, 2: Active partial thromboplastin time.

Table 7-1. Serum biochemical analysis for the male rats in the 13-week repeated oral dose toxicity study (Sex, Male)

\begin{tabular}{|c|c|c|c|c|}
\hline \multicolumn{5}{|c|}{ Summary of Serum Biochemical Tests } \\
\hline \multirow{2}{*}{ Test item (unit) } & \multicolumn{4}{|c|}{ Group (mg/kg/day) } \\
\hline & 0 & 1,250 & 2,500 & 5,000 \\
\hline $\mathrm{AST}^{1}(\mathrm{IU} / \mathrm{L})$ & $103 \pm 38$ & $103 \pm 38$ & $114 \pm 34$ & $133 \pm 76$ \\
\hline $\operatorname{ALT}^{2}(\mathrm{IU} / \mathrm{L})$ & $31 \pm 12$ & $26 \pm 5$ & $31 \pm 6$ & $47 \pm 50$ \\
\hline $\mathrm{ALP}^{3}(\mathrm{IU} / \mathrm{L})$ & $236 \pm 47$ & $263 \pm 44$ & $244 \pm 44$ & $263 \pm 67$ \\
\hline $\mathrm{GGT}^{4}(\mathrm{IU} / \mathrm{L})$ & $0.1 \pm 0.3$ & $0.1 \pm 0.3$ & $0.1 \pm 0.3$ & $0.4 \pm 0.5$ \\
\hline $\mathrm{T}^{-\mathrm{BIL}^{5}}(\mathrm{mg} / \mathrm{dL})$ & $0.04 \pm 0.02$ & $0.05 \pm 0.03$ & $0.04 \pm 0.01$ & $0.06 \pm 0.03$ \\
\hline $\mathrm{BUN}^{6}(\mathrm{mg} / \mathrm{dL})$ & $16.7 \pm 2.7$ & $16.8 \pm 1.9$ & $17.8 \pm 1.3$ & $17.1 \pm 1.5$ \\
\hline $\mathrm{CRE}^{7}(\mathrm{mg} / \mathrm{dL})$ & $0.55 \pm 0.06$ & $0.52 \pm 0.06$ & $0.56 \pm 0.05$ & $0.58 \pm 0.05$ \\
\hline $\mathrm{UA}^{8}(\mathrm{mg} / \mathrm{dL})$ & $2.1 \pm 0.6$ & $2.3 \pm 0.4$ & $2.5 \pm 0.7$ & $2.2 \pm 0.5$ \\
\hline $\mathrm{GLU}^{9}(\mathrm{mg} / \mathrm{dL})$ & $193 \pm 18$ & $187 \pm 25$ & $207 \pm 31$ & $208 \pm 41$ \\
\hline $\mathrm{CHO}^{10}(\mathrm{mg} / \mathrm{dL})$ & $77 \pm 12$ & $71 \pm 20$ & $68 \pm 13$ & $77 \pm 16$ \\
\hline $\mathrm{TG}^{11}(\mathrm{mg} / \mathrm{dL})$ & $57 \pm 24$ & $53 \pm 22$ & $77 \pm 56$ & $59 \pm 8$ \\
\hline $\mathrm{TP}^{12}(\mathrm{~g} / \mathrm{dL})$ & $6.2 \pm 0.2$ & $6.2 \pm 0.2$ & $6.2 \pm 0.3$ & $6.1 \pm 0.3$ \\
\hline $\operatorname{ALB}^{13}(\mathrm{~g} / \mathrm{dL})$ & $2.4 \pm 0.1$ & $2.4 \pm 0.2$ & $2.4 \pm 0.2$ & $2.3 \pm 0.1$ \\
\hline $\mathrm{A} / \mathrm{G}$ ratio $^{14}$ & $0.62 \pm 0.05$ & $0.64 \pm 0.06$ & $0.63 \pm 0.04$ & $0.62 \pm 0.04$ \\
\hline $\mathrm{LDH}^{15}$ (IU/L) & $938 \pm 655$ & $1122 \pm 766$ & $1047 \pm 545$ & $892 \pm 721$ \\
\hline $\mathrm{CPK}^{16}(\mathrm{U} / \mathrm{L})$ & $577 \pm 420$ & $684 \pm 488$ & $663 \pm 318$ & $553 \pm 417$ \\
\hline $\mathrm{Ca}^{17}(\mathrm{mg} / \mathrm{dL})$ & $10.5 \pm 0.2$ & $10.5 \pm 0.3$ & $10.5 \pm 0.3$ & $10.6 \pm 0.3$ \\
\hline $\mathrm{IP}^{18}(\mathrm{mg} / \mathrm{dL})$ & $8.2 \pm 0.6$ & $8.6 \pm 0.6$ & $8.2 \pm 0.6$ & $8.5 \pm 0.5$ \\
\hline $\mathrm{Mg}^{19}(\mathrm{mg} / \mathrm{dL})$ & $2.4 \pm 0.2$ & $2.5 \pm 0.2$ & $2.5 \pm 0.3$ & $2.6 \pm 0.3$ \\
\hline $\mathrm{Na}^{20}(\mathrm{mmol} / \mathrm{L})$ & $139 \pm 4$ & $141 \pm 1$ & $141 \pm 2$ & $141 \pm 1$ \\
\hline $\mathrm{K}^{21}(\mathrm{mmol} / \mathrm{L})$ & $4.7 \pm 0.6$ & $5.0 \pm 0.7$ & $4.8 \pm 0.5$ & $4.9 \pm 0.5$ \\
\hline $\mathrm{Cl}^{22}(\mathrm{mmol} / \mathrm{L})$ & $107 \pm 4$ & $105 \pm 2$ & $104 \pm 2$ & $104 \pm 1$ \\
\hline
\end{tabular}

Mean \pm SD ( $n=10$ per each groups). 1: Aspartate aminotransferase, 2: Alanine aminotransferase, 3: Alkaline phosphatase, 4: Gamma( $\gamma)$-glutamyl transferase, 5: Total bilirubin, 6: Blood urea nitrogen, 7: Creatinine, 8: Uric acid, 9: Glucose, 10: Total cholesterol, 11: Triglycerides, 12: Total protein, 13: Albumin, 14: Albumin/Globulin ratio, 15: Lactate dehydrogenase, 16: Creatine phosphokinase, 17: Calcium, 18: Inorganic phosphorus, 19: Magnesium, 20: Sodium, 21: Potassium, 22: Chloride. 
Table 7-2. Serum biochemical analysis for the female rats in the 13-week repeated oral dose toxicity study (Sex, Female)

\begin{tabular}{|c|c|c|c|c|}
\hline \multicolumn{5}{|c|}{ Summary of Serum Biochemical Tests } \\
\hline \multirow{2}{*}{ Test item (unit) } & \multicolumn{4}{|c|}{ Group (mg/kg/day) } \\
\hline & 0 & 1,250 & 2,500 & 5,000 \\
\hline $\mathrm{AST}^{1}(\mathrm{IU} / \mathrm{L})$ & $112 \pm 20$ & $118 \pm 40$ & $148 \pm 59$ & $134 \pm 64$ \\
\hline $\mathrm{ALT}^{2}(\mathrm{IU} / \mathrm{L})$ & $26 \pm 5$ & $29 \pm 12$ & $40 \pm 20$ & $44 \pm 42$ \\
\hline $\mathrm{ALP}^{3}(\mathrm{IU} / \mathrm{L})$ & $143 \pm 49$ & $142 \pm 31$ & $136 \pm 26$ & $123 \pm 32$ \\
\hline $\mathrm{GGT}^{4}(\mathrm{IU} / \mathrm{L})$ & $0.3 \pm 0.5$ & $0.1 \pm 0.3$ & $0.2 \pm 0.4$ & $0.4 \pm 0.5$ \\
\hline T-BIL 5 (mg/dL) & $0.08 \pm 0.02$ & $0.11 \pm 0.05^{*}$ & $0.08 \pm 0.02$ & $0.08 \pm 0.02$ \\
\hline $\mathrm{BUN}^{6}(\mathrm{mg} / \mathrm{dL})$ & $16.5 \pm 3.9$ & $16.6 \pm 2.6$ & $16.6 \pm 2.4$ & $16.3 \pm 2.2$ \\
\hline $\mathrm{CRE}^{7}(\mathrm{mg} / \mathrm{dL})$ & $0.58 \pm 0.06$ & $0.59 \pm 0.09$ & $0.60 \pm 0.08$ & $0.56 \pm 0.06$ \\
\hline $\mathrm{UA}^{8}(\mathrm{mg} / \mathrm{dL})$ & $1.9 \pm 0.3$ & $1.8 \pm 0.5$ & $1.9 \pm 0.3$ & $2.1 \pm 0.3$ \\
\hline $\mathrm{GLU}^{9}(\mathrm{mg} / \mathrm{dL})$ & $177 \pm 23$ & $186 \pm 26$ & $176 \pm 23$ & $179 \pm 26$ \\
\hline $\mathrm{CHO}^{10}(\mathrm{mg} / \mathrm{dL})$ & $80 \pm 16$ & $81 \pm 11$ & $86 \pm 22$ & $90 \pm 20$ \\
\hline $\mathrm{TG}^{11}(\mathrm{mg} / \mathrm{dL})$ & $34 \pm 25$ & $32 \pm 13$ & $41 \pm 19$ & $40 \pm 18$ \\
\hline $\mathrm{TP}^{12}(\mathrm{~g} / \mathrm{dL})$ & $6.7 \pm 0.4$ & $6.8 \pm 0.3$ & $6.7 \pm 0.4$ & $6.7 \pm 0.5$ \\
\hline $\operatorname{ALB}^{13}(\mathrm{~g} / \mathrm{dL})$ & $2.9 \pm 0.3$ & $3.0 \pm 0.2$ & $2.9 \pm 0.2$ & $2.9 \pm 0.3$ \\
\hline $\mathrm{A} / \mathrm{G}$ ratio $^{14}$ & $0.79 \pm 0.07$ & $0.80 \pm 0.04$ & $0.79 \pm 0.05$ & $0.76 \pm 0.07$ \\
\hline $\mathrm{LDH}^{15}(\mathrm{IU} / \mathrm{L})$ & $1066 \pm 268$ & $1126 \pm 383$ & $1517 \pm 696$ & $1282 \pm 669$ \\
\hline $\mathrm{CPK}^{16}(\mathrm{U} / \mathrm{L})$ & $500 \pm 144$ & $578 \pm 200$ & $733 \pm 362$ & $594 \pm 281$ \\
\hline $\mathrm{Ca}^{17}(\mathrm{mg} / \mathrm{dL})$ & $10.6 \pm 0.8$ & $10.6 \pm 0.5$ & $10.8 \pm 0.5$ & $11.0 \pm 0.2$ \\
\hline $\mathrm{IP}^{18}(\mathrm{mg} / \mathrm{dL})$ & $8.1 \pm 0.5$ & $8.3 \pm 1.2$ & $8.1 \pm 0.5$ & $8.5 \pm 0.8$ \\
\hline $\mathrm{Mg}^{19}(\mathrm{mg} / \mathrm{dL})$ & $2.6 \pm 0.2$ & $2.7 \pm 0.2$ & $2.7 \pm 0.2$ & $2.7 \pm 0.3$ \\
\hline $\mathrm{Na}^{20}(\mathrm{mmol} / \mathrm{L})$ & $140 \pm 1$ & $140 \pm 2$ & $140 \pm 1$ & $139 \pm 2$ \\
\hline $\mathrm{K}^{21}(\mathrm{mmol} / \mathrm{L})$ & $5.0 \pm 0.8$ & $5.0 \pm 0.7$ & $4.9 \pm 0.8$ & $5.8 \pm 0.9$ \\
\hline $\mathrm{Cl}^{22}(\mathrm{mmol} / \mathrm{L})$ & $105 \pm 1$ & $104 \pm 2$ & $103 \pm 1$ & $104 \pm 2$ \\
\hline
\end{tabular}

\footnotetext{
*: Significant difference compared with the control value, $p<0.05$.
}

Mean \pm SD ( $n=10$ per each groups). 1: Aspartate aminotransferase, 2: Alanine aminotransferase, 3: Alkaline phosphatase, 4: Gamma $(\gamma)$-glutamyl transferase, 5: Total bilirubin, 6: Blood urea nitrogen, 7: Creatinine, 8: Uric acid, 9: Glucose, 10: Total cholesterol, 11: Triglycerides, 12: Total protein, 13: Albumin, 14: Albumin/Globulin ratio, 15: Lactate dehydrogenase, 16: Creatine phosphokinase, 17: Calcium, 18: Inorganic phosphorus, 19: Magnesium, 20: Sodium, 21: Potassium, 22: Chloride.

test groups compared with the vehicle control group (Table 7-1, 7-2). Regarding the organ weight, there was no statistically significant weight difference between the test groups and the vehicle group in either sex (Table 8-1, 8-2), however, the relative organ weight of the ovary in the $5,000 \mathrm{mg} /$ $\mathrm{kg}$ test group was statistically significantly decreased compared with the vehicle control $(p<0.05)$ (Table 8-2). However, these results are not considered toxicological effects: because a) the values were within the normal biological range, b) there was no dose-dependency, and c) the results did not correlate with other analysis results.

During histopathological examination of the $5,000 \mathrm{mg} / \mathrm{kg}$ test and vehicle control groups in each sex (Table 9-1, 9-2), focal mononuclear cell infiltration and focal necrosis of the liver, focal basophilic tubule of the kidney, focal tubule degeneration in the outer stripe, hyaline cast in the outer medulla, focal acinar necrosis and mononuclear cell infiltration in the pancreas, ultimobranchial cyst in the thyroid, ectopic thymus, osseous metaplasia, focal hemorrhage and focal mononuclear cell infiltration in the lung, cardiomyopathy of the heart, cyst and focal degradation in the pars distalis of the pituitary gland and lymphocytic cell infiltration in the interstitial prostate were detected in both the vehicle control and $5,000 \mathrm{mg} / \mathrm{kg}$ test groups. In the $1,250 \mathrm{mg} / \mathrm{kg}$ male test group, unilateral hydronephrosis was found in the right kidney of one animal. There were no abnormalities detected in other organs. However, there was no toxicological meaning assigned to the observations listed above because there was no difference in the incidence rate compared to the vehicle control group or because only one or spontaneous lesions were found. Therefore, there were no histopathological abnormal lesions related to the test substance. Other than these findings, there were no abnormalities detected in any other organs.

Skin sensitization test (Buehler method) in Hartley guinea pigs. There were no common symptoms or dead animals during the test period. No tested animals showed significant body weight changes related to the test substance during the experimental period. In the vehicle control and test substance-treated groups, no animals showed visible changes in the test area, while 3 out of 5 animals showed moderate and confluent erythema in the positive control group. The sensitization rate was determined as Grade I 
Table 8-1. Absolute organ weights of the male and female rats in the 13-week repeated oral dose toxicity study

\begin{tabular}{|c|c|c|c|c|c|c|c|c|}
\hline \multicolumn{9}{|c|}{ Summary of Serum Biochemical Tests } \\
\hline \multirow{3}{*}{ Test item (unit) } & \multicolumn{4}{|c|}{ Sex: Male } & \multicolumn{4}{|c|}{ Sex: Female } \\
\hline & \multicolumn{4}{|c|}{ Group (mg/kg/day) } & \multicolumn{4}{|c|}{ Group (mg/kg/day) } \\
\hline & 0 & 1,250 & 2,500 & 5,000 & 0 & 1,250 & 2,500 & 5,000 \\
\hline Body weight & $520.04 \pm 21.84$ & $542.81 \pm 35.47$ & $543.59 \pm 61.64$ & $540.01 \pm 36.72$ & $282.83 \pm 31.72$ & $285.49 \pm 24.63$ & $290.81 \pm 23.22$ & $301.59 \pm 18.20$ \\
\hline Testis/Ovary (Lt.) & $1.7932 \pm 0.1414$ & $1.7340 \pm 0.1550$ & $1.7463 \pm 0.1658$ & $1.7658 \pm 0.0598$ & $0.0436 \pm 0.0063$ & $0.0451 \pm 0.0040$ & $0.0477 \pm 0.0107$ & $0.0387 \pm 0.0069$ \\
\hline Testis/Ovary (Rt.) & $1.7177 \pm 0.4190$ & $1.7580 \pm 0.1678$ & $1.7692 \pm 0.1666$ & $1.6606 \pm 0.3447$ & $0.0464 \pm 0.0102$ & $0.0459 \pm 0.0089$ & $0.0441 \pm 0.0088$ & $0.0456 \pm 0.0044$ \\
\hline Prostate/Uterus & $0.8153 \pm 0.1616$ & $0.8264 \pm 0.2454$ & $0.9535 \pm 0.2716$ & $0.7558 \pm 0.1766$ & $0.6548 \pm 0.1760$ & $0.8147 \pm 0.3198$ & $0.6326 \pm 0.2119$ & $0.6197 \pm 0.2019$ \\
\hline Spleen & $0.8915 \pm 0.0994$ & $0.9394 \pm 0.1354$ & $1.0031 \pm 0.2659$ & $0.9338 \pm 0.1332$ & $0.5728 \pm 0.0733$ & $0.6015 \pm 0.1272$ & $0.5832 \pm 0.0701$ & $0.5987 \pm 0.0665$ \\
\hline Liver & $12.6002 \pm 0.7069$ & $13.5555 \pm 1.6576$ & $14.2476 \pm 2.2318$ & $13.6109 \pm 1.3072$ & $6.7039 \pm 2.4853$ & $7.6403 \pm 0.9585$ & $7.4266 \pm 0.5667$ & $7.8332 \pm 0.6983$ \\
\hline Adrenal gland (Lt.) & $0.0302 \pm 0.0043$ & $0.0293 \pm 0.0058$ & $0.0303 \pm 0.0069$ & $0.0332 \pm 0.0036$ & $0.0399 \pm 0.0103$ & $0.0392 \pm 0.0063$ & $0.0383 \pm 0.0040$ & $0.0346 \pm 0.0053$ \\
\hline Adrenal gland (Rt.) & $0.0296 \pm 0.0048$ & $0.0266 \pm 0.0062$ & $0.0316 \pm 0.0046$ & $0.0317 \pm 0.0051$ & $0.0381 \pm 0.0075$ & $0.0375 \pm 0.0083$ & $0.0374 \pm 0.0052$ & $0.0331 \pm 0.0042$ \\
\hline Kidney (Lt.) & $1.4340 \pm 0.1185$ & $1.5134 \pm 0.1526$ & $1.5143 \pm 0.0958$ & $1.4956 \pm 0.1086$ & $0.8936 \pm 0.0772$ & $0.9149 \pm 0.0963$ & $0.9129 \pm 0.0749$ & $0.9442 \pm 0.0438$ \\
\hline Kidney (Rt.) & $1.4824 \pm 0.1666$ & $1.5407 \pm 0.1378$ & $1.5480 \pm 0.1056$ & $1.5460 \pm 0.0830$ & $0.9316 \pm 0.0764$ & $0.9653 \pm 0.0915$ & $0.9320 \pm 0.0782$ & $0.9826 \pm 0.0467$ \\
\hline Heart & $1.5542 \pm 0.1220$ & $1.5367 \pm 0.1588$ & $1.5086 \pm 0.1259$ & $1.6398 \pm 0.2300$ & $1.0427 \pm 0.1449$ & $1.0482 \pm 0.0694$ & $1.0197 \pm 0.0832$ & $1.0527 \pm 0.0607$ \\
\hline Lung & $1.7434 \pm 0.1185$ & $1.7470 \pm 0.1215$ & $1.9604 \pm 0.6487$ & $1.8720 \pm 0.1715$ & $1.3729 \pm 0.0964$ & $1.3541 \pm 0.1200$ & $1.3798 \pm 0.1581$ & $1.3866 \pm 0.0736$ \\
\hline Brain & $2.1403 \pm 0.0489$ & $2.1539 \pm 0.0991$ & $2.1223 \pm 0.0833$ & $2.1289 \pm 0.1127$ & $1.9564 \pm 0.0746$ & $1.9688 \pm 0.0899$ & $1.9784 \pm 0.0813$ & $2.0015 \pm 0.0936$ \\
\hline Pituitary & $0.0124 \pm 0.0024$ & $0.0116 \pm 0.0022$ & $0.0124 \pm 0.0038$ & $0.0141 \pm 0.0019$ & $0.0182 \pm 0.0042$ & $0.0178 \pm 0.0029$ & $0.0163 \pm 0.0030$ & $0.0192 \pm 0.0045$ \\
\hline Thymus & $0.2989 \pm 0.0616$ & $0.3095 \pm 0.0723$ & $0.3006 \pm 0.0632$ & $0.2828 \pm 0.0571$ & $0.2636 \pm 0.0987$ & $0.2651 \pm 0.0804$ & $0.2443 \pm 0.0480$ & $0.2799 \pm 0.0753$ \\
\hline
\end{tabular}

Mean \pm SD ( $n=10$ per each groups).

Table 8-2. Relative organ weights of the male and female rats in the 13-week repeated oral dose toxicity study

\begin{tabular}{|c|c|c|c|c|c|c|c|c|}
\hline \multicolumn{9}{|c|}{ Summary of Serum Biochemical Tests } \\
\hline \multirow{3}{*}{ Test item (unit) } & \multicolumn{4}{|c|}{ Sex: Male } & \multicolumn{4}{|c|}{ Sex: Female } \\
\hline & \multicolumn{4}{|c|}{ Group (mg/kg/day) } & \multicolumn{4}{|c|}{ Group (mg/kg/day) } \\
\hline & 0 & 1,250 & 2,500 & 5,000 & 0 & 1,250 & 2,500 & 5,000 \\
\hline Testis/Ovary (Lt.) & $0.3452 \pm 0.0293$ & $0.3205 \pm 0.0328$ & $0.3251 \pm 0.0482$ & $0.3286 \pm 0.0292$ & $0.0155 \pm 0.0024$ & $0.0159 \pm 0.0020$ & $0.0164 \pm 0.0033$ & $0.0128 \pm 0.0022^{*}$ \\
\hline Testis/Ovary (Rt.) & $0.3314 \pm 0.0820$ & $0.3247 \pm 0.0324$ & $0.3297 \pm 0.0520$ & $0.3105 \pm 0.0735$ & $0.0163 \pm 0.0025$ & $0.0162 \pm 0.0035$ & $0.0152 \pm 0.0029$ & $0.0152 \pm 0.0016$ \\
\hline Prostate/Uterus & $0.1564 \pm 0.0286$ & $0.1548 \pm 0.0535$ & $0.1755 \pm 0.0412$ & $0.1399 \pm 0.0305$ & $0.2339 \pm 0.0690$ & $0.2893 \pm 0.1206$ & $0.2186 \pm 0.0746$ & $0.2059 \pm 0.0671$ \\
\hline Spleen & $0.1717 \pm 0.0204$ & $0.1730 \pm 0.0214$ & $0.1850 \pm 0.0495$ & $0.1726 \pm 0.0179$ & $0.2031 \pm 0.0201$ & $0.2096 \pm 0.0329$ & $0.2017 \pm 0.0283$ & $0.1988 \pm 0.0216$ \\
\hline Liver & $2.4240 \pm 0.1183$ & $2.4929 \pm 0.1908$ & $2.6126 \pm 0.1617$ & $2.5173 \pm 0.1107$ & $2.3963 \pm 0.8688$ & $2.6703 \pm 0.1552$ & $2.5605 \pm 0.1872$ & $2.5956 \pm 0.1269$ \\
\hline Adrenal gland (Lt.) & $0.0058 \pm 0.0008$ & $0.0054 \pm 0.0009$ & $0.0057 \pm 0.0016$ & $0.0062 \pm 0.0009$ & $0.0143 \pm 0.0040$ & $0.0138 \pm 0.0022$ & $0.0132 \pm 0.0013$ & $0.0115 \pm 0.0019$ \\
\hline Adrenal gland (Rt.) & $0.0057 \pm 0.0008$ & $0.0049 \pm 0.0011$ & $0.0059 \pm 0.0012$ & $0.0059 \pm 0.0011$ & $0.0136 \pm 0.0027$ & $0.0132 \pm 0.0029$ & $0.0129 \pm 0.0020$ & $0.0110 \pm 0.0017$ \\
\hline Kidney (Lt.) & $0.2760 \pm 0.0235$ & $0.2791 \pm 0.0249$ & $0.2804 \pm 0.0218$ & $0.2780 \pm 0.0254$ & $0.3185 \pm 0.0343$ & $0.3214 \pm 0.0309$ & $0.3147 \pm 0.0238$ & $0.3140 \pm 0.0219$ \\
\hline Kidney (Rt.) & $0.2853 \pm 0.0324$ & $0.2843 \pm 0.0236$ & $0.2869 \pm 0.0269$ & $0.2873 \pm 0.0221$ & $0.3320 \pm 0.0355$ & $0.3393 \pm 0.0323$ & $0.3211 \pm 0.0230$ & $0.3267 \pm 0.0208$ \\
\hline Heart & $0.2992 \pm 0.0255$ & $0.2833 \pm 0.0252$ & $0.2791 \pm 0.0228$ & $0.3038 \pm 0.0375$ & $0.3685 \pm 0.0288$ & $0.3687 \pm 0.0289$ & $0.3515 \pm 0.0272$ & $0.3504 \pm 0.0322$ \\
\hline Lung & $0.3360 \pm 0.0295$ & $0.3223 \pm 0.0182$ & $0.3611 \pm 0.1107$ & $0.3467 \pm 0.0218$ & $0.4896 \pm 0.0525$ & $0.4751 \pm 0.0284$ & $0.4763 \pm 0.0569$ & $0.4603 \pm 0.0187$ \\
\hline Brain & $0.4121 \pm 0.0160$ & $0.3985 \pm 0.0339$ & $0.3948 \pm 0.0449$ & $0.3956 \pm 0.0298$ & $0.6993 \pm 0.0799$ & $0.6935 \pm 0.0582$ & $0.6834 \pm 0.0503$ & $0.6666 \pm 0.0609$ \\
\hline Pituitary & $0.0024 \pm 0.0005$ & $0.0021 \pm 0.0003$ & $0.0023 \pm 0.0008$ & $0.0026 \pm 0.0003$ & $0.0065 \pm 0.0017$ & $0.0063 \pm 0.0012$ & $0.0056 \pm 0.0011$ & $0.0064 \pm 0.0014$ \\
\hline Thymus & $0.0576 \pm 0.0126$ & $0.0572 \pm 0.0139$ & $0.0550 \pm 0.0073$ & $0.0522 \pm 0.0083$ & $0.0917 \pm 0.0295$ & $0.0923 \pm 0.0255$ & $0.0843 \pm 0.0162$ & $0.0922 \pm 0.0219$ \\
\hline
\end{tabular}

Mean \pm SD $\left(n=10\right.$ per each groups). ${ }^{*}:$ Significant difference compared with the control value, $p<0.05$. 
Table 9-1. Histopathological findings in the male rats in the 13-week repeated oral dose toxicity study (Sex, Male)

\begin{tabular}{|c|c|c|c|c|c|c|c|c|}
\hline \multicolumn{9}{|c|}{ Summary of Serum Biochemical Tests } \\
\hline \multirow{3}{*}{ Organs } & \multirow{3}{*}{ Sings } & & \multicolumn{6}{|c|}{ Group (mg/kg/day) } \\
\hline & & & \multicolumn{2}{|c|}{0} & \multicolumn{2}{|c|}{1,250} & \multicolumn{2}{|c|}{5,000} \\
\hline & & & $\mathrm{N}$ & $\%$ & $\mathrm{~N}$ & $\%$ & $\mathrm{~N}$ & $\%$ \\
\hline \multirow{4}{*}{ Liver } & No remarkable lesions & & $6 / 10$ & 60 & & & $8 / 10$ & 80 \\
\hline & Remarkable lesions & & $4 / 10$ & 40 & & & $2 / 10$ & 20 \\
\hline & - Cell infiltration, mononuclear, focal & \pm & $1 / 10$ & 10 & & & $1 / 10$ & 10 \\
\hline & - Necrosis, focal & \pm & $3 / 10$ & 30 & & & $1 / 10$ & 10 \\
\hline \multirow{5}{*}{ Kidney } & No remarkable lesions & & $8 / 10$ & 80 & $0 / 1$ & 0 & $10 / 10$ & 100 \\
\hline & Remarkable lesions & & $2 / 10$ & 20 & $1 / 1$ & 100 & $0 / 10$ & 0 \\
\hline & - Basophilic tubule, focal & \pm & $1 / 10$ & 10 & $0 / 1$ & 0 & $0 / 10$ & 0 \\
\hline & - Degeneration, tubule, focal, outer stripe & \pm & $1 / 10$ & 10 & $0 / 1$ & 0 & $0 / 10$ & 0 \\
\hline & - Hydronephrosis, unilateral & \pm & $0 / 10$ & 0 & $1 / 1$ & 100 & $0 / 10$ & 0 \\
\hline \multirow{3}{*}{ Pancrease } & No remarkable lesions & & $10 / 10$ & 100 & & & $9 / 10$ & 90 \\
\hline & Remarkable lesions & & $0 / 10$ & 0 & & & $1 / 10$ & 10 \\
\hline & - Necrosis, focal, acinar & \pm & $0 / 10$ & 0 & & & $1 / 10$ & 10 \\
\hline \multirow{4}{*}{ Thyroid } & No remarkable lesions & & $7 / 10$ & 70 & & & $10 / 10$ & 100 \\
\hline & Remarkable lesions & & $3 / 10$ & 30 & & & $0 / 10$ & 0 \\
\hline & - Ultimobranchial cyst & \pm & $2 / 10$ & 20 & & & $0 / 10$ & 0 \\
\hline & - Ectopic thymus & \pm & $2 / 10$ & 20 & & & $0 / 10$ & 0 \\
\hline \multirow{4}{*}{ Lung } & No remarkable lesions & & $10 / 10$ & 100 & & & $8 / 10$ & 80 \\
\hline & Remarkable lesions & & $0 / 10$ & 0 & & & $2 / 10$ & 20 \\
\hline & - Hemorrhage, focal, perivascular & \pm & $0 / 10$ & 0 & & & $1 / 10$ & 10 \\
\hline & - Osseous metaplasia, alveolar & \pm & $0 / 10$ & 0 & & & $1 / 10$ & 10 \\
\hline \multirow{3}{*}{ Heart } & No remarkable lesions & & $9 / 10$ & 90 & & & $10 / 10$ & 100 \\
\hline & Remarkable lesions & & $1 / 10$ & 10 & & & $0 / 10$ & 0 \\
\hline & - Cardiomyopathy & \pm & $1 / 10$ & 10 & & & $0 / 10$ & 0 \\
\hline \multirow{4}{*}{ Pituitary } & No remarkable lesions & & $9 / 10$ & 90 & & & $7 / 9$ & 78 \\
\hline & Remarkable lesions & & $1 / 10$ & 10 & & & $2 / 9$ & 22 \\
\hline & - Cyst, pars distalis & \pm & $1 / 10$ & 10 & & & $1 / 9$ & 11 \\
\hline & - Degeneration, focal, pars distalis & \pm & $0 / 10$ & 0 & & & $1 / 9$ & 11 \\
\hline \multirow{3}{*}{ Prostate } & No remarkable lesions & & $9 / 10$ & 90 & & & $9 / 10$ & 90 \\
\hline & Remarkable lesions & & $1 / 10$ & 10 & & & $1 / 10$ & 10 \\
\hline & - Cell infiltration, lymphocytic, interstitial & \pm & $1 / 10$ & 10 & & & $1 / 10$ & 10 \\
\hline
\end{tabular}

$\mathrm{N}$ : Number of animals with the signs/Number of examined animals, \pm : Minimal.

(very weak) for the vehicle control, Grade II for the test substance-treated group, and Grade II (moderate) for the positive control group at 24 and $48 \mathrm{hr}$ after removal of the challenge patch (Fig. 3). Therefore, the final sensitization rate of cricket powder was determined to be $0 \%$ and was evaluated as "very weak", which corresponds with Grade I.

\section{DISCUSSION}

The crickets G. bimaculatus, teleogryllus occipitalis, and Teleogryllus mitratus are commonly consumed as food in Asia (8). Particularly, Acheta domesticus, which is known as a house cricket, is also reared and commonly consumed as food and approximately 20,000 cricket farms exist in Thailand (9). There are several reasons why the edible insect industry is increasing. With respect to health, the environment, and livelihood, the edible insect business is likely to have advantages over the livestock business in the future (10). Cricket rearing requires fewer water supplies than cattle rearing, with fewer animal welfare issues, and also poses a low risk of transmitting zoonotic infections (11). Studies have shown that insects are healthy nutritious alternatives to poultry or bovine meat, being rich in protein, good fat, and minerals (12). Moreover, insects also emit significantly less greenhouse gas compared to other livestock (13). As an example, ammonia emission rates from insects are much lower than conventional livestock (14).

Our study demonstrates that crickets cause no toxicological issues and can serve as a food source. The major components are protein, fat, and fiber. Cricket is high in several 
Table 9-2. Histopathological findings in the female rats in the 13-week repeated oral dose toxicity study (Sex, Female)

\begin{tabular}{|c|c|c|c|c|c|c|}
\hline \multicolumn{7}{|c|}{ Summary of Serum Biochemical Tests } \\
\hline \multirow{3}{*}{ Organs } & \multirow{3}{*}{ Sings } & & \multicolumn{4}{|c|}{ Group (mg/kg/day) } \\
\hline & & & \multirow{2}{*}{$\begin{array}{c}0 \\
\mathrm{~N}\end{array}$} & \multicolumn{3}{|c|}{5,000} \\
\hline & & & & $\%$ & $\mathrm{~N}$ & $\%$ \\
\hline \multirow{3}{*}{ Liver } & No remarkable lesions & & $10 / 10$ & 100 & $10 / 10$ & 100 \\
\hline & Remarkable lesions & & $0 / 10$ & 0 & $1 / 10$ & 10 \\
\hline & - Cell infiltration, mononuclear, focal & \pm & $0 / 10$ & 0 & $1 / 10$ & 10 \\
\hline \multirow{3}{*}{ Kidney } & No remarkable lesions & & $10 / 10$ & 100 & $9 / 10$ & 90 \\
\hline & Remarkable lesions & & $0 / 10$ & 0 & $1 / 10$ & 10 \\
\hline & - Hyaline cast, outer medulla & \pm & $0 / 10$ & 0 & $1 / 10$ & 10 \\
\hline \multirow{3}{*}{ Pancreas } & No remarkable lesions & & $9 / 10$ & 90 & $10 / 10$ & 100 \\
\hline & Remarkable lesions & & $1 / 10$ & 10 & $0 / 10$ & 0 \\
\hline & - Cell infiltration, mononuclear, focal, acinar & \pm & $1 / 10$ & 10 & $0 / 10$ & 0 \\
\hline \multirow{3}{*}{ Lung } & No remarkable lesions & & $9 / 10$ & 90 & $9 / 10$ & 90 \\
\hline & Remarkable lesions & & $1 / 10$ & 10 & $1 / 10$ & 10 \\
\hline & - Cell infiltration, mononuclear, focal & \pm & $1 / 10$ & 10 & $1 / 10$ & 10 \\
\hline
\end{tabular}

$\mathrm{N}$ : Number of animals with the signs/Number of examined animals, \pm : Minimal.

(A)

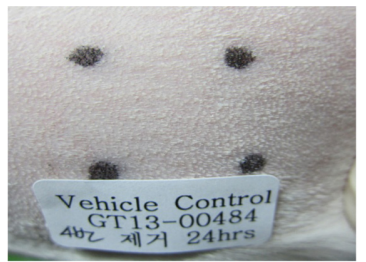

(B)

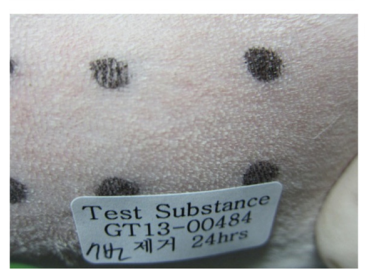

(C)

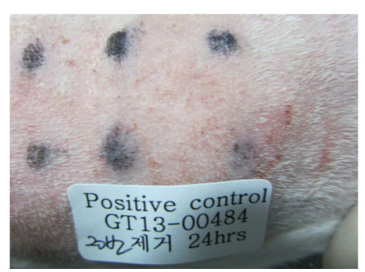

(D)

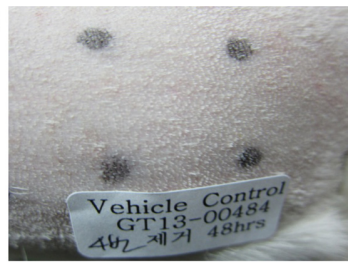

(E)

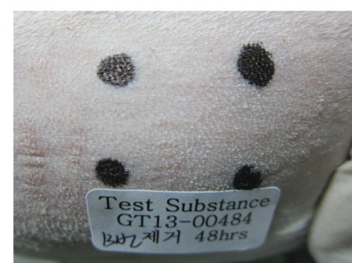

(F)

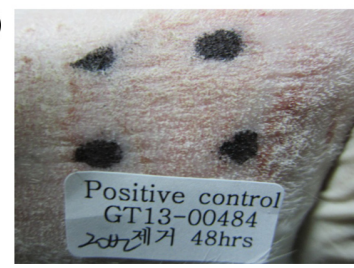

Fig. 3. Skin reaction of cricket powder at 24 and $48 \mathrm{hr}$ after removal of the challenge patch. Representative pictures of skin reaction of vehicle control after (A) $24 \mathrm{hr}$, (B) $48 \mathrm{hr}$ after challenge patch removal, cricket powder after (C) $24 \mathrm{hr}$, (D) $48 \mathrm{hr}$ after challenge patch removal, and positive control after $(E)$ $24 \mathrm{hr}$, (F) $48 \mathrm{hr}$ after challenge patch removal. No skin irritation has been shown in the cricket powder treated group.

amino acids, unsaturated fatty acids and micronutrients such as mineral and vitamins. Micronutrients influence the nutritional quality of food (15). Micronutrient deficiencies prevalent in developing countries are causing major adverse health consequences such as impairments in immune sys- tem, growth, reproduction, mental or physical development (16). The micronutrient content of crickets comprised of a relatively high amount of zinc, iron, manganese and copper in our experiment as well as in other studies and consumption of crickets generally elevates nutritional content (17). Iron deficiency is a common and widespread nutritional disorder (18). In 2004, WHO reported that there were 270,000 deaths caused by iron deficiency anemia: 45, 31, 7 and $3 \%$ mortality rates were found in Southeast Asia, Africa, United States, and Europe, respectively (19). Moreover, health issues resulting from anemia such as cognitive and impaired physical development as well as child deaths are prevalent in low- and middle- income countries (20). Zinc also plays an important role in biochemical pathways in the human body including the reproductive, immune and central nervous systems. Zinc deficiency may manifest as acne, alopecia, night blindness, pneumonia, diarrhea, anorexia nervosa, physical disorders or hypogonadism (21-25). Because cricket has a high content of iron and zinc, it can reduce food shortage in developing countries while at the same time maintaining the body iron content by including cricket powder in the daily diet (26). Essential fatty acids such as alpha linoleic acid and linoleic acid cannot be synthesized within the human body (16). According to the FAO/WHO recommendations from 1994, the consumption of unsaturated fatty acid is emphasized during the developmental phase in infants and children (27). Insects including G. bimaculatus have been found to be easily digestible because they contain high quality proteins and essential amino acids (15). They may serve as alternatives to meat and improve the nutritional quality of food in the future. Given the high contents of essential fatty acids, minerals, protein and amino acids in cricket, further evaluations of more edible insect 
species are warranted.

Under the conditions described for the administration of cricket powder, there were no toxicological effects on body weight change, food consumption, ophthalmoscopy, urinalysis, plasma coagulation, hematological or serum biochemical analysis, organ weight, or histopathological analysis. In addition, a target organ was not detected. Animals tolerated up to and including $5,000 \mathrm{mg} / \mathrm{kg} /$ day for both sexes of rats without adverse effects in a 13-week repeated oral toxicity study. Also, it was determined that there was no skin hypersensitivity reaction to the cricket. These results collectively suggest that the acceptable daily intake (ADI) is up to 50 $\mathrm{mg}$ per $\mathrm{kg}$ of body weight. Due to the high contents of protein, unsaturated fatty acids, amino acids and minerals, crickets can be used as an excellent food source, resolving the scarcity of food, in addition to being a health supplement in the future.

\section{ACKNOWLEDGEMENTS}

This study was supported by the Rural Development Administration for the joint research project (Project number: PJ00982703). The authors declare no conflict of interest.

\section{REFERENCES}

1. Ahn, M.Y., Lee, Y.W., Ryu, K.S., Lee, H.S., Kim, I.S., Kim, J.W. and Lim, S.S. (2004) Effects of water and methanol extracts of cricket (Gryllus bimaculatus) on alcohol metabolism. Korean J. Pharmacogn., 35, 175-178.

2. Ahn, M.Y., Hwang, J.S. and Yun, E.Y. (2015) Gene expression profiling of glycosaminoglycan drived from G. bimaculatus in high fat dieted rat. FASEB J., 29, LB152.

3. Ahn, M.Y., Han, J.W., Hwang, J.S., Yun, E.Y. and Lee, B.M. (2014) Anti-inflammatory effect of glycosaminoglycan derived from Gryllus bimaculatus (a type of cricket, insect) on adjuvant-treated chronic arthritis rat model. J. Toxicol. Environ. Health Part A, 77, 1332-1345.

4. Kim, I.S., Ahn, M.Y., Ryu, K.S. and Lee, B.M. (2002) Acute oral toxicity of G. bimaculatus in rats. J. Toxicol. Pub. Health, 18, 397-400.

5. OECD (1992) Test No. 406: Skin sensitisation in OECD Guidelines for the Testing of Chemicals, Section 4. Available from: http://www.oecd-ilibrary.org/environment/test-no-406-skinsensitisation_9789264070660-en (Accessed Dec. 27, 2012).

6. OECD (1998) Test No. 408: Repeated Dose 90-Day Oral Toxicity Study in Rodents in OECD Guidelines for the Testing of Chemicals, Section 4. Available from: http://www.oecd-ilibrary.org/environment/test-no-408-repeated-dose-90-day-oraltoxicity-study-in-rodents 9789264070707-en (Accessed Dec. 27, 2012).

7. Basketter, D.A. and Gerberick, G.F. (1996) An interlaboratory evaluation of the Buehler test for the identification and classification of skin sensitizers. Contact Derm., 35, 146-151.

8. Ramos-Elorduy, J. (2002) Edible insects of chiapas, Mexico. Ecol. Food Nutr., 41, 271-299.
9. Hanboonsong, Y., Jamjanya, T. and Durst, P.B. (2013) Sixlegged livestock: edible insect farming, collection and marketing in Thailand, FAO, Bangkok, p. 5.

10. Rumpold, B.A. and Schlüter, O.K. (2013) Nutritional composition and safety aspects of edible insects. Mol. Nutr. Food Res., 57, 802-823.

11. van Huis, A. (2013) Potential of insects as food and feed in assuring food security. Annu. Rev. Entomol., 58, 563-583.

12. Wang, D., Bai, Y.Y., Li, J.H. and Zhang, C.X. (2004) Nutritional value of the field cricket (Gryllus testaceus Walker). Insect Sci., 11, 275-283.

13. Oonincx, D.G., van Itterbeeck, J., Heetkamp, M.J., van den Brand, H., van Loon, J.J. and van Huis, A. (2010) An exploration on greenhouse gas and ammonia production by insect species suitable for animal or human consumption. PLoS One, 5, e14445..

14. Steinfeld, H., Gerber, P., Wassenaar, T., Castel, V., Rosales, M. and Haan, C.D. (2006) Livestock's long shadow: environmental issues and options, FAO, Rome, pp. 82-83, 151-152.

15. Kinyuru, J.N., Mogendi, J.B., Riwa, C.A. and Ndung'u, N.W. (2015) Edible insects - a novel source of essential nutrients for human diet: Learning from traditional knowledge. Animal Frontiers, 5, 14-19.

16. Michaelsen, K.F., Hoppe, C., Roos, N., Kaestel, P., Stougaard, M., Lauritzen, L., Mølgaard, C., Girma, T. and Friis, H. (2009) Choice of foods and ingredients for moderately malnourished children 6 months to 5 years of age. Food Nutr. Bull., 30, S343-S404.

17. Huis, V.A., Itterbeeck, V.J., Klunder, H., Mertens, E., Halloran, A., Muir, G. and Vantomme, P. (2013) Edible insects: future prospects for food and feed security, FAO, Rome, pp. 67-68.

18. McLean, E., Cogswell, M., Egli, I., Wojdyla, D. and Benoist, B. (2009) Worldwide prevalence of anaemia, WHO vitamin and mineral nutrition information system, 1993-2005. Public Health Nutr., 12, 444-454.

19. Pasricha, S.R., Drakesmith, H., Black, J., Hipgrave, D. and Biggs, B.A. (2013) Control of iron deficiency anemia in lowand middle-income countries. Blood, 121, 2607-2617.

20. Kassebaum, N.J., Jasrasaria, R., Naghavi, M., Wulf, S.K., Johns, N., Lozano, R., Regan, M., Weatherall, D., Chou, D.P., Eisele, T.P., Flaxman, S.R., Pullan, R.L., Brooker, S.J. and Murray, C.J. (2014) A systematic analysis of global anemia burden from 1990 to 2010. Blood, 123, 615-624.

21. Yamada, T., Alpers, D.H., Kalloo, A.N., Kaplowitz, N., Owyang, C. and Powell, D.W. (2009) Textbook of gastroenterology (5th edition), Wiley-blackwell pub, Chichester, pp. 17-18.

22. Kumar, P. and Clark, M.L. (2012) Kumar \& Clark's clinical medicine (8th edition), Elsevier Saunders, Edinburgh, pp. 587-588.

23. Lassi, Z.S., Haider, B.A. and Bhutta, Z.A. (2010) Zinc supplementation for the prevention of pneumonia in children aged 2 months to 59 months. Cochrane Database Syst. Rev., (12), CD005978.

24. Suzuki, H., Asakawa, A., Li, J.B., Tsai, M., Amitani, H., Ohinata, K., Komai, M. and Inui, A. (2011) Zinc as an appetite stimulator-the possible role of zinc in the progression of diseases such as cachexia and sarcopenia. Recent Pat. Food Nutr. Agric., 3, 226-231. 
25. Swardfager, W., Herrmann, N., Mazereeuw, G., Goldberger, K., Harimoto, T. and Lanctôt, K.L. (2013) Zinc in depression: a meta-analysis. Biol. Psychiatry, 74, 872-878.

26. Muller, O. and Krawinkel, M. (2005) Malnutrition and health in developing countries. CMAJ, 173, 279-286.
27. World Health Organization and Food and Agriculture Organization of the United Nations (1994) Fats and oils in human nutrition: report of a joint expert consultation. FAO and WHO Expert Consultation, Rome, Available from: http://www.fao.org/ docrep/V4700E/V4700E00.htm (Accessed Sep. 12, 2012). 